\title{
OBSERVATION OF THE ASKARYAN EFFECT IN ICE WITH THE ANITA EXPERIMENT
}

\section{A THESIS SUBMITTED TO THE GRADUATE DIVISION OF THE UNIVERSTTY OF HAWAI'I IN PARTIAL FULFILLMENT OF THE REQUIREMENTS FOR THE DEGREE OF \\ MASTER OF SCIENCE}

IN

PHYSICS

AUGUST 2007

\author{
By \\ Richard Jeffrey Kowalski
}

Thesis Committee:

Peter W. Gorham, Chairperson

Gary S. Varner

John G. Learned 
We certify that we have read this thesis and that, in our opinion, it is satisfactory in scope and quality as a thesis for the degree of Master of Science in Physics.

THESIS COMMITTEE
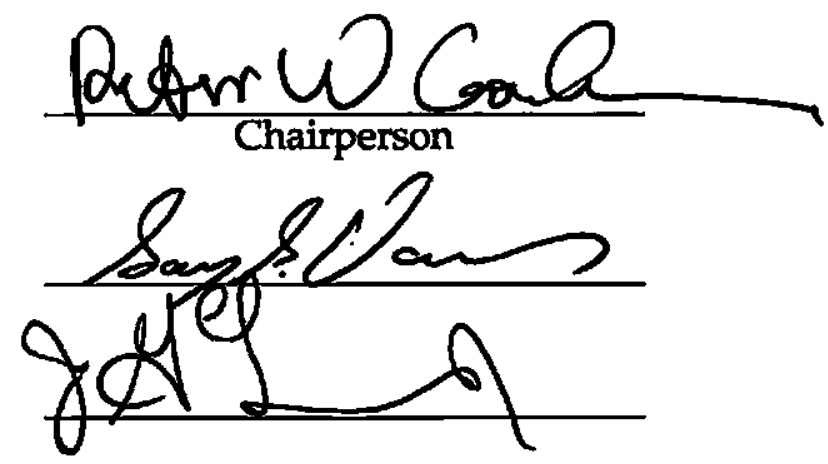


\section{Copyright 2007}

by

R. Jeffrey Kowalski 
To Mom and Dad,

Debbie and Rick Kowalski 


\section{Acknowledgements}

In the 3 years I have spent working on the ANITA project at the University of Hawai'i at Mãnoa, I have gained support from many individuals and institutions. I'll begin by expressing my gratitude to the following:

Professor Peter W. Gorham has been my thesis advisor and the principal investigator for the ANITA experiment. From his knowledge of RF system design, data analysis, laboratory and experimental conduct, and astrophysics, he has given me many of the essential tools needed to be a successful experimentalist. In working with him both in $\mathrm{UH}$ labs and in the field (SLAC T461 \& T486 at Stanford University and A-142-M in Antarc(ica), I have gained the most valuable experience in my educational career. Many thanks to Dr. Gorham for all of his teachings over the years.

Professor Gary S. Varner for his ongoing help with firmware development, hardware integration, and data analysis. His leading examples shown while working with him in the field have improved my work tremendously and have given me the guidance to continue with this research.

ANITA project engineer, Marc Rosen, for his ongoing guidance during mechanical integration of ANITA subsystems and John G. Learned for his experience and motivation in particle astrophysics.

Members of the ANITA collaboration for playing a significant role during the course of this research and will continue to be colleagues in the future. In particular, I would like to acknowledge: Kim Palladino (OSU), David Saltzberg (UCLA), James Beatty (OSU), Paul Dowkowntt (WashU), Dana Braun (WashU), Ryan Nichol (OSU), David Goldstein (UCI), Chuck Naudet (JPL), Kurt Liewer (JPL), Bob Binns (WashL), Amy Connolly $(U C L A)$, Michael DuVernois (UM), and Jiwoo Nam (UCI).

Our support staff during multiple instrument integrations has been tremendous. Melvin Matsunaga and Roy Tom have made numerous contributions toward design and fabrication of ANITA hardware. I'd also like to thank Jan Bruce for help during all of the travel toward this research, and Jeff Griskevich for his cooperation at UCI integrations and for his excellent facility management.

I have also had the pleasure of working with various undergraduate, graduate students and post-doctoral researchers at the University of Hawai'i at Mãnoa. Specifically, I'd like to thank: Larry Ruckman for his continuous help with electronics and firmware development, Jim Kennedy, Hannibal Starbuck, Christian Miki, Bryce Jacobson, Andres 
Romero-Wolf, Mike Hadmack for always having guidance toward developing Python code, Shige Matsuno, Predrag Miocinovic, Jason Link, and Nikolai Lehtinen.

During the weeks at SLAC, I had the opportunity to interact many talented individuals. A few of them are: Dieter Walz for his knowledge of the accelerator facilities and physics; Clive Field for helping during the construction of the ice target and for getting a few last measurements for me; Richard Iverson for his knowledge of accelerator beam control; Carl Hudspeth for his cooperation while working in ESA and his foresight in producing the ice target.

The support crew at CSBF supercedes their expectations. The crew chief for ANITA, Victor Davison, along with multiple individuals from their science support team aided in the success of the instrument and were essential during mechanical integration of the payload.

Having the opportunity to travel and work in Antarctica was a great experience and would not have been achievable without support from USAP, Raytheon Polar Services, and NSF.

Finally, funding and financial support is a vital element when conducting research at an academic institution. This research would not have been made possible without the support from NASA under grant \# NAG5-5387 and the Department of Energy Office of Science High Energy Physics Division. Lastly, special thanks to the SLAC Experiment Facilities Department. 


\begin{abstract}
First hypothesized by Gurgen Askaryan in the 1960's and later confirmed in 2001 at SLAC (Stanford Linear Accelerator Center), radio Cherenkov detection techniques are possible in the ultra-high energy regime $\left(10^{18} \rightarrow 10^{22} \mathrm{eV}\right)$ while observing electromagnetic cascades in dielectric media. This method of detection has now moved into the field of neutrino astrophysics. Recently, the interest in using ice as a dielectric medium to observe coherent microwave Cherenkov pulses from ultra-high energy neutrino induced particle showers has grown considerably with advances from experiments such as RICE, FORTE, and ANITA-lite. ANITA (ANtarctic Impulsive Transient Antenna), is a radio telescope designed to exploit this effect while looking for UHE neutrino interactions in Antarctic ice. In June 2006, ANITA observed these highly coherent radio impulses in SLAC's ESA (End Station A) with $28.5 \mathrm{GeV}$ electrons interacting with a 7.5 tonne ice target to produce the EM shower. These first measurements of the Askaryan effect in ice were consistent with shower and electrodynamics simulations for ice and provided a clear indication that the radiation is coherent over the $200-1200 \mathrm{MHz}$ frequency window. In addition to the ANITA payload in SLAC's ESA, four log-period dipole array antennas, two monocone antennas, and one high frequency gain horn (nominally 2.6-3.95 GHz) recorded impulsive events emanating from the ice target. I report on further analysis of coherent radio Cherenkov impulses using the standard gain horn and demonstrate that the results are fully consistent with theoretical expectations.
\end{abstract}




\section{Contents}

Acknowledgements $\quad$ v

Abstract vii

List of Tables $\quad x$

List of Figures $\quad$ xi

List of Acronyms xiv

1 Introduction 1

2 Neutrino Astrophysics 3

2.1 GZK Limit . . . . . . . . . . . . . . . . . . . 4

2.1.1 Experimental Support for the GZK Cutoff . . . . . . . . . . 6

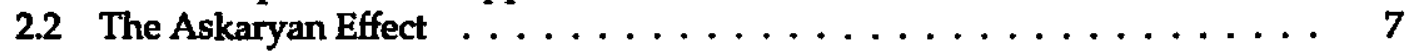

2.2.1 Efforts in UHE $\nu$ Detection $\ldots \ldots \ldots \ldots . \ldots \ldots$

3 The ANITA Instrument 11

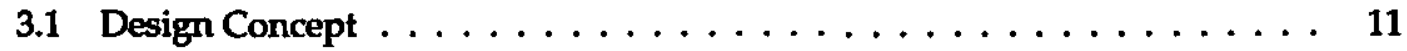

3.2 Overview of ANITA Payload $\ldots \ldots \ldots \ldots \ldots \ldots \ldots \ldots \ldots \ldots$

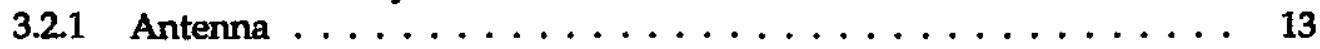

3.2 .2 Gondola . . . . . . . . . . . . . . . . . . . . 14

3.3 Data Acquisition System, Subsystems and Integrated Hardware . . . . . . 15

3.3.1 Power Consumption . . . . . . . . . . . . . . . 15

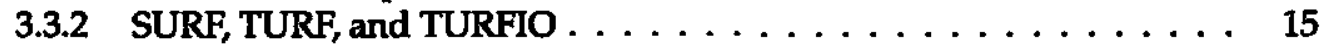

3.3 .3 GPS . . . . . . . . . . . . . . . . . 17

3.3.4 Calibration Pulser $\ldots \ldots \ldots \ldots \ldots$

3.3.5 External Sensors . . . . . . . . . . . . . . . 18

4 Electron Beam Experiment at the Stanford Linear Accelerator 20

4.1 Experimental Setup . . . . . . . . . . . . . . . . . 20

4.1 .1 Beam Current . . . . . . . . . . . . . . . . 21

4.1.2 RF Monitor and External Antennas .............. 21

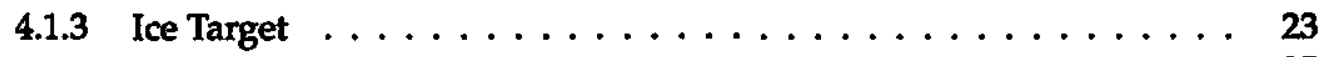

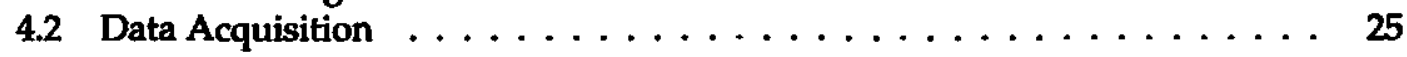

viii 
4.2.1 Dipole Calibration . . . . . . . . . . . . . . . . 27

4.2.2 Gain Horn Calibration . . . . . . . . . . . . . . . . . . . 29

4.3 Electric Field Reconstruction . . . . . . . . . . . . . . . . . 35

5 Conclusion 38

A SLAC T486 Ice Target 39

B Bases, Camps, \& Research Stations on the Antarctic Continent 43

$\begin{array}{ll}\text { Bibliography } & 47\end{array}$ 


\section{List of Tables}

$3.1 \phi$-sector assignments for the ANITA payload . . . . . . . . . . . . . 15

3.2 Triggering and sampling capabilities of the ANITA experiment [1]. . . . . 17

4.1 A summary of the additional antenna used in T486 with their mapping to oscilloscope. The top half of the table shows the initial setup for data acquisition and the bottom half denotes the setup after 06/20/06 2am PDT. . . 27 


\section{List of Figures}

2.1 Cosmic Ray Energy Spectrum [2]: The flux for lower energy cosmic rays (yellow) are associated with solar cosmic ray sources, intermediate energies (blue) with sources from our local galaxy, and higher energies (purple) with sources of extra-galactic origin. . . . . . . . . . . . . . .

2.2 UHE Neutrino Interaction in Ice: Graphical representation of a UHE $\nu$ collision with an atom in ice (not to scale). The neutrino, travelling faster than $c$, collides with an atom or molecule in the ice producing an electromagnetic shower. At $\theta_{C} \simeq 56^{\circ}\left(\cos \theta_{C} \simeq 1 / n_{i c e}\right)$, the RF emission propagates to the surface where it refracts and is received by the ANITA payload. . . . . .

3.1 Conceptual View of the ANITA Experiment: At a float altitude of $\sim 37 \mathrm{~km}$, the ANITA payload is tethered from a NASA LDB filled with $\mathrm{He}$ to a volume near 2 million $\mathrm{m}^{3}$ and a diameter of $\sim 175 \mathrm{~m}$. Due to the low RF attenuation of Antarctic ice, the ANITA receiver system is able to detect EMP's emanating from UHE $\nu$ 's interactions in the ice. . . . . . . . . . . . .

3.2 The ANITA Payload: 32 Seavey dual-polarized, quad-ridge horn antennas, 8 monitor antennas, 2 GPS systems, instrument enclosures, and 2 PV arrays for ANITA and CSBF electronics respectively. . . . . . . . . . . . .

3.3 RF Receiver System: Schematic view of the front end RF system. Incoming radio waves are captured with Seavey quad-ridge horns. Signals from 2 antennas are fed through an RFCM where the signal is bandpass filtered and amplified by $80 \mathrm{~dB}(40 \mathrm{~dB}+40 \mathrm{~dB})$. The outgoing signal is then sent to the ANITA instrument. . . . . . . . . . . . . . . . . . . . 14

3.4 Firmware Flow Diagram: Control logic for ANITA's data acquisition system. 16

4.1 SLAC T486 Experimental Setup: Rendered view of the ANITA payload with the $28.5 \mathrm{GeV}$ electron beam at SLAC's ESA. . . . . . . . . . . . . . . 21

4.2 SLAC T486 Beam Current: Beam Current vs. time showing the number of electron bunches per unit energy per unit time. The data sets came from 2 individual monitoring toroids which span the duration of the experiment. .

4.3 Diagram showing the relative position of the dipole antenna to the beam pipe and the external RF absorber. . . . . . . . . . . . . 22

-4.4 Antenna positions for T486: Top left and right show the positions of the gain horn, LPDA's, Monocones, PCB LPDA's, and the ANITA instrument relative to the ice target. . . . . . . . . . . . . . . 
4.5 Photographs of SLAC T486 Ice Target: (Top left) Front view of ice target with $\sim 8^{\circ}$ slope. (Top right) Front view of ice target with foam insulation cover and cooling ducts shown. This was the configuration for the majority of runs during the experiment. (Bottom left) Close-up showing the surface roughness of the ice target. (Bottom right) Top view of ice target with measured surface roughness grid from appendix A (not to scale). . . . . . . . .

4.6 Schematic view of the Ice Target's Surface Roughness: The above describes the physical layout of the ice target for T486 (not to scale). . . . . . . .

4.7 Plot of T486 ice surface. All values are in inches. Note the pronounced change in slope at $\approx 140^{\prime \prime} \approx 3.5 \mathrm{~m} . \ldots \ldots \ldots \ldots \ldots$

4.8 Left: Region A and Right: Region B of the T486 ice target. The fitted plane (red) to the measured grid points (blue) is shown revealing two distinct inclination angles $-8.4^{\circ}$ and $2.6^{\circ}$ respectively. All dimensions are in inches here. . . . . . . . . . . . . . . . . . . . . . 26

4.9 Waveforms with the respective power spectrum from run 126 , event 888 . . .

4.10 Run 102 peak voltage distributions for the dipole antenna and gain horn which is normalized to the mean of the dipole peak distribution. For run 102, the dipole distribution has $\mu=0.2273$ and $\sigma=0.0379$, which provides a normalized horn distribution with $\mu=\mathbf{5 . 2 6 0 2}$ and $\sigma=0.9537$. All units are in relative volts. . . . . . . . . . . . . . . . . .

4.11 Detected pulse power as a function of shower energy for 19 runs during T486. The dipole antenna, depicted in figure 4.3 , measured the transition radiation exiting the beam pipe at an angle of $56.5^{\circ}$ from the beam axis. The quadratic rise with beam energy is characteristic of coherent radiation. . . .

$4.12 \mathrm{E} \& \mathrm{H}$ - Fields of low, mid, and high-bands for Standard Gain Horn (Scientific-Atlanta, Inc.). . . . . . . . . . . . . . . . . . . .

4.13 Left: E-plane gain and Right: H-plane gain for low- (green), mid- (blue), and high-bands (orange) of the horn antenna. The beam pattern narrows significantly with higher frequencies suggesting the gain should increase

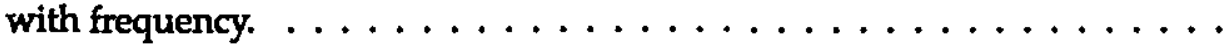

4.14 Half-power-beam-width as a function of frequency for the Standard Gain Horn showing the main lobe of the beam pattem narrowing at higher frequencies. . . . . . . . . . . . . . . . . 32

4.15 Directivity (top) and gain (bottom) as a function of frequency. . . . . . . . 33

4.16 Effective area (top) and effective height (bottom) for the Standard Gain Horn as a function of frequency. . . . . . . . . . . . . . 34

4.17 Detected pulse power as a function of shower energy for the Standard Gain Horn. . . . . . . . . . . . . . . . . . . . .

4.18 (Top) Waveform received by the hom antenna in run 102 with the corresponding power spectrum (bottom). . . . . . . . . . . 
4.19 Electric field strength vs. frequency of radio Cherenkov radiation from the T486 experiment for several different antennas used, including the expected theoretical curve $[3,4]$. The lower frequency data was borrowed from Gorham et al. [5] measured with the ANITA instrument. The high frequency data came from this work with the Standard Gain Horn which illustrates the roll-off of the Cherenkov radiation from electromagnetic showers in ice with frequency. . . . . . . . . . . . . . . . . . 


\section{List of Acronyms}

ADC Analog-to-Digital Converter

AGASA Akeno Giant Air Shower Array

AGN Active Galactic Nuclei

AMANDA Antarctic Muon And Neutrino Detector Array

ANITA ANtarctic Impulsive Transient Antenna

ASIC Application-Specific Integrated Circuit

AVZ Alvarez-Muñiz-Vázquez-Zas

AWS Automated-Weather-Station

CMB Cosmic Microwave Background

CR Cosmic Ray

CSBF Columbia Scientific Balloon Facility

DAQ Data AcQuisition

EAS Extensive Air Shower

EMI ElectroMagnetic Interference

EMP ElectroMagetic Pulse

ESA End Station A

EUSO Extreme Universe Space Observatory

FDTD Finite-Difference-Time-Domain 
FORTE Fast On-orbit Recorder of Transient Events

GLUE Goldstone Lunar Ultra-high energy neutrino Experiment

GPS Global Positioning System

GRB Gamma-Ray Burst

GZK Greisen-Zatsepin-Kuzmin

HPBW Half-Power-Beam-Width

ITASE International Trans-Antarctic Scientific Expedition

JPL Jet Propulsion Laboratory

LABRADOR Large Analog Bandwidth Recorder And Digitizer with Ordered Readout

LCP Left-Circular Polarized

LDB Long Duration Balloon

LNA Low Noise Amplifier

LOS Line-Of-Sight

LPDA Log-Periodic Dipole Array

LPM Landau-Pomeranchuck-Migdal

NASA National Aeronautics and Space Administration

NOAA National Oceanic and Atmospheric Administration

NSF National Science Foundation

OWL OverWhelmingly Large

PCB Printed Circuit Board

PPS Pulse Per Second

PV PhotoVoltaics

RCP Right-Circular Polarized

XV 
RICE Radio Ice Cherenkov Experiment

RF Radio Frequency

RFCM Radio Frequency Conditioning Module

SHORT SURF High-Occupancy RF Trigger

SIP Support Instrumentation Package

SLAC Stanford Linear Accelerator Center

SMA Sub-Miniature A

SURF Sampling Unit for Radio Frequencies

TDRSS Tracking and Data Relay Satellite System

TIGER Trans-Iron Galactic Element Recorder

TIR Total Internal Reflection

TTL Transistor-Transistor Logic

TURF Trigger Unit for Radio Frequencies

TURFIO Trigger Unit for Radio Frequencies InputOutput

UHE Ultra-High Energy

UHECR Ultra-High Energy Cosmic Rays

USAP United States Antarctic Program

ZHS Zas-Halzen-Stanev 


\section{Chapter 1}

\section{Introduction}

Our understanding of the origin and nature of ultra-high energy cosmic rays (UHECR) and their accompanying neutrinos remains as one of the fundamental problems of experimental particle astrophysics. Highest energy cosmic rays have long been suspected to be from extragalactic sources since the Milky Way Galaxy cannot magnetically contain and accelerate them to the energies we observe. We still do not have a confirmed astrophysical source of these particles nor do we understand fully how they are accelerated to the Earth. The observations of particles with energies above $10^{20} \mathrm{eV}$ with experiments such as HiRes and Auger have provided strong experimental support for the Greisen-Zatsepin-Kuzmin cutoff $[6,7]$ and confidence in the existence of neutrinos reaching energies in the EeV scale ${ }^{1}$. Detection of such neutrinos would provide new physical insight into our understanding of active galactic nuclei (AGN), gamma ray bursts, supernovae, etc. since neutrinos pass effortlessly through space to the Earth. Ultra-high energy neutrinos, therefore, enable us to peer into the environment of the early universe and discover possible source evolutions since neutrinos will point back to their origin.

The progress in capabilities of using coherent radio Cherenkov emission to detect high energy particles has provided a possible alternative for discovering ultra-high energy (UHE) neutrinos. Since Askaryan's early prediction that electromagnetic showers in dense media would produce an excess charge that generates coherent Cherenkov pulses in radio frequencies $[8,9]$, many new detectors have arisen to exploit the effect of neutrino induced showers with the Moon [10], Greenland ice [11], and Antarctic ice [12, 13, 14] as the target medium. The validity of this method was first emphasized in a detailed study combining electrodynamics with electromagnetic shower simulations in ice [3]. Furthermore, a care-

\footnotetext{
${ }^{1} 1 \mathrm{EeV}=10^{18} \mathrm{eV}$
} 
ful treatment of the detection of radio pulses from high energy showers has been examined under the classic descriptions of Fraunhofer [4] and Fresnel [15] zones.

Chapter 2 discusses the developments in the field of neutrino astrophysics with an emphasis on the GZK cutoff (2.1, 2.1.1) and the Askaryan Effect ( 2.2, 2.2.1) which the ANITA experiment relies on.

In chapter 3, I provide an overview of the ANITA experiment. Here I outline the gondola design ( 3.1) with a brief overview of the payload (3.2). Special attention is directed toward the ANITA data acquisition system (3.3) since the vast majority of my work with the ANITA collaboration was spent on the development of the RF and data acquisition electronics.

The first measurement of the Askaryan effect in ice, SLAC T486, is discussed in chapter 4 outlining the experiment 4.1, data acquisition 4.2, and analyses performed (4.2.1, 4.2.2, 4.3). I finish with a discussion of the results from SLAC T486 in chapter 5. 


\section{Chapter 2}

\section{Neutrino Astrophysics}

Neutrinos, they are small.

They have no charge and no mass

And do not interact at all.

The earth is just a silly ball

To them, through which they simply pass,

Like dustmaids down a drafty hall

Or photons through a sheet of glass ...

by John Updike [16]

Today, we know that this Updike's 1960 poem has a fundamental flaw - neutrinos do have mass albeit tiny! How massive are they? Do they oscillate? Are neutrinos the key to unfolding the mystery of dark matter and missing energy in the universe?

In 1930, Wolfgang Pauli hypothesized that a neutral particle (zero charge) could carry the missing energy in nuclear $\beta$-decays [17]. Three years later, Enrico Fermi named this particle the neutrino $(\nu)$. It wasn't until 1956 where Frederick Reines and Clyde Cowon found the first experimental evidence for the electron (anti-) neutrino using a large $400 \mathrm{~L}$ water and cadmium chloride $\left(\mathrm{CdCl}_{2}\right)$ detector $12 \mathrm{~m}$ underground [18]. In this experiment, Reines and Cowon looked at the inverse $\beta$-decay $\left(\nu+p \rightarrow n+e^{+}\right)$and measured the gamma ray photons produced from positrons annihilating with electrons using photomultiplier tubes. However, due to the nature of the weak interaction and the fact that neutrinos carry no electric charge, the probability of an interaction involving neutrinos is extremely small making detection very difficult. 
In the 1960 's, the Homestake experiment began operation by Ray Davies to hunt for solar neutrinos produced in the Sun's core from thermonuclear reactions that power the Sun. The flux of electron-neutrinos observed was a factor of one third less than the standard solar developed by John Bahcall and others and "solar neutrino problem" arose.

We now know from the $Z$-boson $\left(Z^{0}\right)$ decay width, that there must be 3 light neutrinos in the Standard Model - one for each fermion family. The neutrinos produced are detected in weak interactions as flavours: electron-neutrino $\left(\nu_{e}\right)$, muon-neutrino $\left(\nu_{\mu}\right)$, and the tau-neutrino $\left(\nu_{\tau}\right)$. It seems that the most convincing explanation for the missing solar neutrino flux stems from the $\nu_{e}$ oscillating into the other neutrino flavours. In order for oscillation to occur, neutrinos must have mass and neutrino flavours must mix [19]. The implications have developed an entirely new field of neutrino astrophysics which will continue to unfold fundamental neutrino properties and peer into the physics of the early Universe.

Today, we know that neutrinos are the most penetrating form of radiation in the universe, and can pass through magnetic fields or intense radiation fields without difficulty at all energies. Direct measurements of high-energy neutrinos would provide confirmation of cosmic acceleration of hadrons (baryons and mesons) from higher energy particles such as protons, electrons, helium nuclei, and heavier nuclei (cosmic rays) and would illuminate the nature of their source since neutrinos are essentially unaffected during transit.

\subsection{GZK Limit}

The main motivation to search for cosmological high-energy neutrino sources is derived from the theory that the cosmic ray energy spectrum extends to energies greater than $10^{20} \mathrm{eV}$. If cosmic rays follow an energy spectrum given by:

$$
\frac{d N}{d E_{p}} \propto E_{p}^{-2}
$$

we should see a steep cutoff occurring near $10^{20} \mathrm{eV}$ due to the rapid decrease in particle flux. The cosmic ray energy spectrum does not follow a perfect power law given by equation 2.1.1. The true spectrum has intrinsic properties shown in figure 2.1 where the power law varies considerably. 


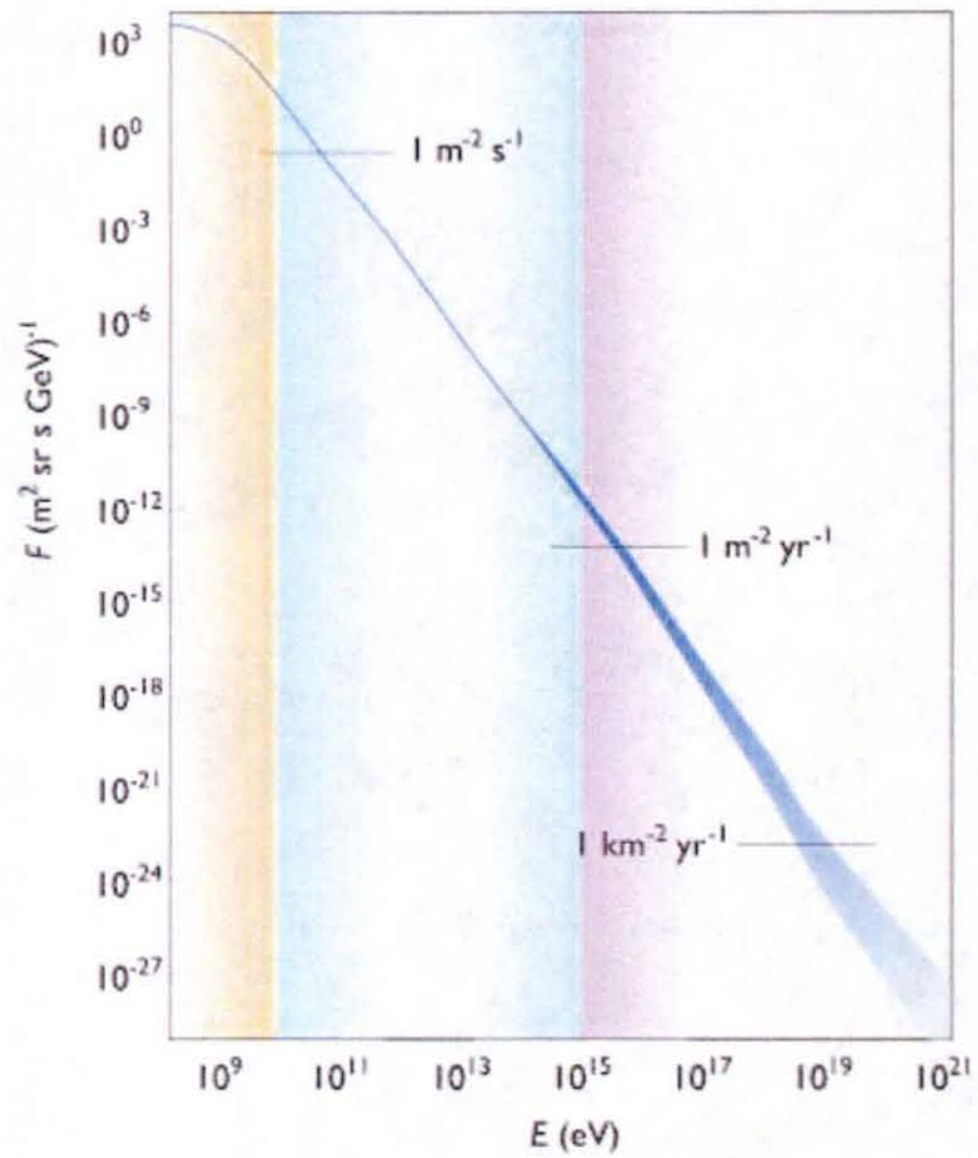

Figure 2.1: Cosmic Ray Energy Spectrum [2]: The flux for lower energy cosmic rays (yellow) are associated with solar cosmic ray sources, intermediate energies (blue) with sources from our local galaxy, and higher energies (purple) with sources of extra-galactic origin. 
Above $10^{9} \mathrm{eV}$, the CR energy spectrum can be described by a series of power laws, with the flux falling about 3 orders of magnitude for each decade increase in energy. At the "knee" ( $\left.10^{15.5} \mathrm{eV}\right)$, the spectrum steepens from $\mathrm{E}^{-2.7}$ to $\mathrm{E}^{-3.0}$ which is still not consistently explained after being discovered over 40 years ago [20]. Above $\sim 10^{17.7} \mathrm{eV}$ ("the dip"), the spectrum steepens further to $\mathrm{E}^{-3.3}$ and then flattens out to $\mathrm{E}^{-2.7}$ near the "an$\mathrm{kle}^{\prime \prime}$ at $\sim 10^{18.5} \mathrm{eV}$. Sources above $\sim 3 \times 10^{18.5} \mathrm{eV}$ are most likely extra-Galactic protons [21] produced from naturally occurring particle accelerators (i.e. AGN's, BL Lac's, GRB's, etc.).

In 1966, Greisen, Zatsepin, and Kuzmin [6,7] predicted that the flux of these UHE protons would be reduced via the process:

$$
p+\gamma_{2.7 K} \rightarrow \Delta^{*} \rightarrow n+\pi^{ \pm}
$$

where the decay chain of the $\pi^{ \pm}$leads to the flux of high energy neutrinos ("GZK $\nu$ flux") which are observable to the visible edge of the universe, whereas CR's are limited to our local supercluster $(\leq 40 \mathrm{Mpc})^{2}[14]$.

\subsubsection{Experimental Support for the GZK Cutoff}

When a high energy cosmic ray hits the upper atmosphere $(\sim 20 \mathrm{~km})$, a jet of particles is created that continues to travel in the same direction as the cosmic ray. As particles in the jet collide with nuclei of oxygen and nitrogen in the air, secondary cosmic rays are produced which can yield over a million particles which can be detected by a large telescope array on Earth. The detection of Extensive Air Showers (EAS) has proven to be a successful means of measuring the the energy spectra of ultra-high energy cosmic rays which extends beyond $10^{20} \mathrm{eV}$. Where does GZK suppression begin?

Currently, the best sources for the highest energy cosmic rays has come from five experimental collaborations: AGASA [22], Fly's Eye [23], Haverah Park [24], HiRes [25], and Yakutsk [26]. The results from the five different experiments has a two-fold conclusion suggesting that all data with energies below $10^{20} \mathrm{eV}$ is in good agreement while all data consistent with a GZK suppression points above $10^{20} \mathrm{eV}$ with the exception of the AGASA collaboration [27]. The results from HiRes and AGASA have created an inconsistency in the literature for experimental evidence that supports a GZK cutoff that is still under debate.

\footnotetext{
${ }^{1} \pi^{+} \rightarrow \mu^{+}+\nu_{\mu} ; \pi^{-} \rightarrow \mu^{-}+\bar{\nu}_{\mu} ; \pi^{+} \rightarrow e^{+}+\nu_{e}, \pi^{-} \rightarrow e^{-}+\bar{\nu}_{e}$

${ }^{2} 1 \mathrm{Mpc}=3.0857 \times 10^{22} \mathrm{~m}$
} 
The next major experiment to probe the energy and spectrum of UHECR's is the Pierre Auger Cosmic Ray Observatory (AUGER). Built in western Argentina, this largescale surface array utilizes two methods for detection of UHECR's (both air fluorescence telescopes and water Cherenkov methods) making it a "hybrid detector." Although the Auger Observatory is only $\mathbf{8 5} \%$ complete, results have already been announced confirming the presence of the ankle and steepening of the $C R$ spectrum near $\sim 10^{19} \mathrm{eV}[28]$.

Neutrino astronomy has recently become a major player in the ability to confirm GZK suppression. Existing experiments such as ANITA, IceCube, RICE, etc. and next generation experiments being planned (EUSO, OWL, SalSA, $X$-RICE) may confirm the existence of GZK neutrinos which will compliment and expand AUGER observations. The study of UHE $\nu$ 's has an advantage of allowing for observation into the interior of objects whereas protons can only show the surface since interactions during propagation obscure the early Universe from direct observation [29].

\subsection{The Askaryan Effect}

During the development of a high-energy electromagnetic particle cascade in normal matter, photons will knock electrons from the material into the shower. This process is known as Compton scattering $\left(\gamma+e_{\text {atom }}^{-} \rightarrow \gamma+e^{-}\right)$and dominates the likelihood for asymmetry of electrons over positrons. Some positrons will annihilate in flight $\left(e^{+}+e_{\text {atom }}^{-} \rightarrow \gamma \gamma\right)$ terminating positron trajectories while other high-energy process such as Bhabha scattering $\left(e^{+}+e_{\text {atom }}^{-} \rightarrow e^{+}+e^{-}\right)$and Moller scattering $\left(e^{-}+e_{\text {atom }}^{-} \rightarrow e^{-}+e^{-}\right)$add more electrons to the shower but do not change the total energy electron bunch propagating through the media. The cumulative effect develops a negative charge asymmetry of 20-30\%. The charge excess [3] is approximated by:

$$
Q=\frac{N_{e^{-}}-N_{e^{+}}}{N_{e^{-}}+N_{e^{+}}}
$$

which induces an electric current. Since the dimensions of the clump of charged particles are small compared to the wavelength of radio waves, the electromagnetic shower radiates coherent radio Cherenkov radiation whose power is proportional to the square of the net charge in the shower. The net charge in the shower is proportional to the primary energy so the radiated power scales quadratically with the shower energy $\left(P_{R F} \propto E^{2}\right)$. This effect 
was first hypothesized by Gurgen Askaryan in 1962 [8, 9] and is depicted in figure 2.2 where ice is the dielectric medium.

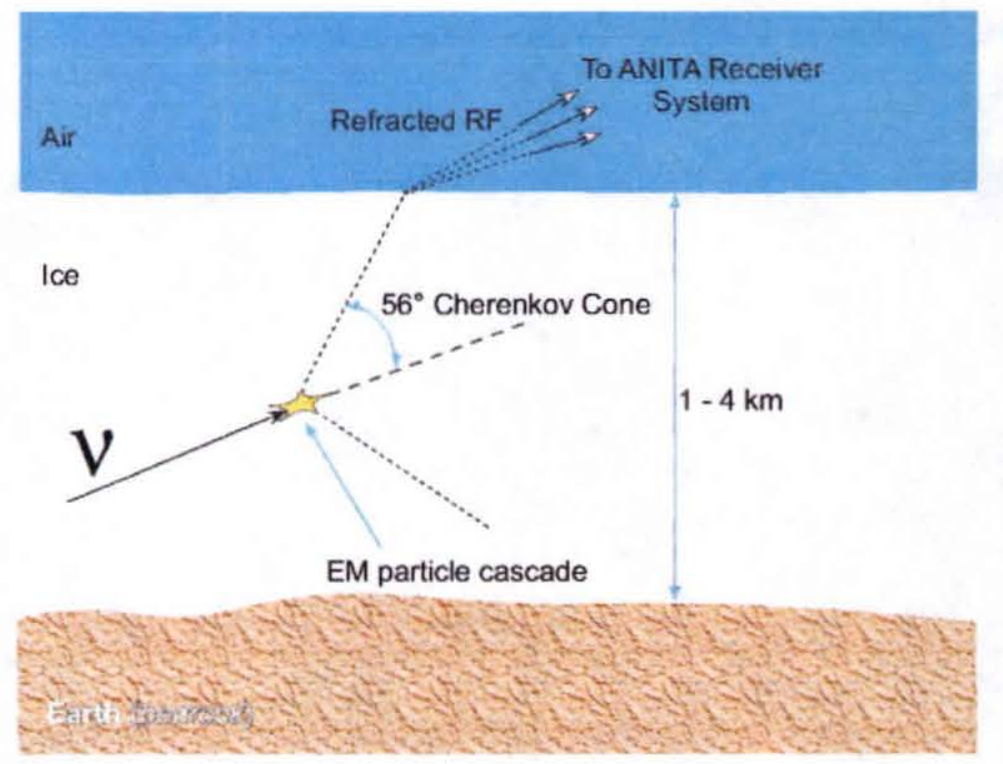

Figure 2.2: UHE Neutrino Interaction in Ice: Graphical representation of a UHE $\nu$ collision with an atom in ice (not to scale). The neutrino, travelling faster than $c$, collides with an atom or molecule in the ice producing an electromagnetic shower. At $\theta_{C} \simeq 56^{\circ}\left(\cos \theta_{C} \simeq\right.$ $1 / n_{\text {ice }}$ ), the RF emission propagates to the surface where it refracts and is received by the ANITA payload.

In the early 1990's, Zas, Halzen, and Stanev [3] (ZHS) provided the first detailed simulations of the electromagnetic pulses generated by shower electrons in ice. Here, an empirical formula for the electric field pulse spectrum, $\mathrm{E}(\nu)$, was derived giving the result:

$$
R|\mathbf{E}(\nu)|=1.1 \times 10^{-7} \frac{\mathcal{E}_{\text {shower }}}{1 \mathrm{TeV}} \frac{\nu}{\nu_{0}} \frac{1}{1+0.4\left(\nu / \nu_{0}\right)^{2}} \times \mathrm{e}^{-\left(\theta-\theta_{\mathbf{C}}\right)^{2} / 2 \Delta \theta} \quad \mathrm{V} \mathrm{MHz}^{-1},
$$

where $\mathrm{R}$ is the distance to the observation point in ice, $\mathcal{E}_{\text {shower }}$ is the shower energy, $\nu$ is the electromagnetic wave frequency, $\nu_{0}=500 \mathrm{MHz}, \theta_{C}$ is the Cherenkov angle in ice, and $\Delta \theta$ $=2.4^{\circ} \nu / \nu_{0}$. A more recent result from Alvarez-Muñiz, Vázquez, and Zas [4] (AVZ) gives a new parametrization for the frequency spectrum in the Cherenkov direction with finer resolution than $\mathrm{ZHS}$ and is valid up to $\sim 5 \mathrm{GHz}$. This is given by:

$$
R\left|\mathbf{E}\left(\nu, \mathbf{R}, \theta_{\mathbf{C}}\right)\right| \simeq 2.53 \times 10^{-7} \frac{\mathcal{E}_{\text {shower }}}{1 \mathrm{TeV}} \frac{\nu}{\nu_{0}} \frac{1}{1+\left(\nu / \nu_{0}\right)^{1.44}} \quad \mathrm{~V} \mathrm{MHz}{ }^{-1},
$$


where $\nu_{0}$ is now $1.15 \mathrm{GHz}$ for ice.

Zas et al. also discuss the dependence of radio frequency with the width of the Cherenkov cone and show that the two are inversely proportional [3] which is consistent with slit diffraction if one considers the dimensions of the antenna's feed-point to be much smaller than the wavelength of electromagnetic radiation. Since the width of the Cherenkov cone broadens at low frequencies, equation 2.2.2 becomes inaccurate and it an analytic estimate is necessary. Lehtinen et al. [11] show that by assuming the shower is a point charge moving at the speed of light in the $\hat{z}$ direction with current ${ }^{3}$ $J_{z}(\mathbf{r}, \mathbf{t})=\mathbf{c q}(\mathbf{z}) \delta(\mathbf{r}-\mathbf{c t \hat { z }})$, the electromagnetic cascade can be approximated by a Gaussian shower profile $q(z)=Q e^{-z^{2} / 2 L^{2}}$ where $\mathrm{L}$ is the length of the electromagnetic shower and $Q$ is the maximum attained charge excess given by equation 2.2.1. The estimate for the electric field pulse spectrum becomes:

$$
R|\mathbf{E}(\nu)|=\sqrt{2 \pi} \mu \mu_{0} \mathbf{Q L} \nu \sin \theta \mathbf{e}^{-(\mathbf{k L})^{2}(\cos \theta-1 / \mathbf{n})^{2} / 2}
$$

where $\mu=1$ for typical dielectrics, $\mu_{0}$ is the permeability of free space, and $\theta$ is the polar angle around the shower axis. It should be mentioned here, that according to the Landau-Pomeranchuck-Migdal (LPM) effect, equations 2.2.2 and 2.2.4 are not valid when electromagnetic showers are started by particles with high energies $\left(\mathcal{E}>\mathcal{E}_{L P M}\right.$, where $\mathcal{E}_{L P M}=2.4 \times 10^{15} \mathrm{eV}$ ). At these high energies, a small fraction of the electromagnetic shower displays characteristic elongation which reduces the width of the Cherenkov cone. Extensive simulations have shown this effect for EeV hadronic showers in ice [30]. However, in this analysis, we need not consider LPM effects since the measured Cherenkov emission was induced by $28.5 \mathrm{GeV}$ electrons discussed in section 4.1.1.

\subsubsection{Efforts in UHE $\nu$ Detection}

Detection of UHE $\nu$ events is difficult due to their extreme rarity. For neutrinos with fluxes comparable to the ultra-high energy cosmic ray flux at $10^{21} \mathrm{eV}$ shown in figure 2.1, detection volumes of order $10^{6} \mathrm{~km}^{3}$ sr are necessary to achieve useful sensitivity [31]. Large scale optical Cherenkov detectors such as AMANDA [13] and IceCube [32] have successfully demonstrated the means of detecting Cherenkov radiation from neutrino interactions $>10^{12} \mathrm{eV}$ with Antarctic ice as a target medium but utilized $\sim \mathrm{km}^{3} \mathrm{sr}$

\footnotetext{
${ }^{3} J_{x}=J_{y}=0$
} 
scale volumetric apertures. Evolving the detection regime to energies from $10^{17}-10^{21} \mathrm{eV}$ involves a new method which utilizes the detection of radio Cherenkov radiation.

Nearly 40 years after Askaryan's prediction, the first laboratory tests of the Askaryan effect were performed using silica sand [33] and later with rock salt [34]. These results provided firm confirmation of coherence of RF emission within a dielectric medium. Furthermore, other analysis has shown that most of the energy from an Askaryan pulse arrives with 0.1 ns [35]. Prior to these measurements, in the late 1990's the Radio Ice Cherenkov Experiment (RICE) [12] began operation at the South Pole to test the validity of the Askaryan effect. Since mid-2000, the Goldstone Lunar Ultra-high energy neutrino Experiment (GLUE) [10] became an ongoing experiment to probe $10^{21} \mathrm{eV}$ energy neutrinos using the regolith in the lunar surface as a dielectric medium. The ability to detect coherent pulsed radio emission from lunar distances provoked the interest to develop satellite and balloonpayload experiments to observe large volumes of ice existing on Earth. The Fast On-orbit Recorder of Transient Events (FORTE) using dual-polarization antennas with a frequency range of 30 to $300 \mathrm{MHz}$ observed the Greenland ice sheet and set the first experimental limit on neutrino fluxes in the $10^{22}-10^{25} \mathrm{eV}$ energy regime [11]. In 2003, the balloon-borne experiment, ANITA-lite, set the best limits on neutrino fluxes above $10^{19.5} \mathrm{eV}$ [14] after flying a primarily engineering test over the Antarctic ice sheet with the TIGER payload.

The success of the ANITA-lite mission has provided the support to develop a fullscale ANITA detector to improve the sensitivity of constraining neutrino fluxes in the UHE region and make the first discovery of a UHE neutrino of extragalactic origin. 


\section{Chapter 3}

\section{The ANITA Instrument}

With the utilization of the Askaryan Effect, the Antarctic Impulsive Transient Antenna will seek to discover UHE neutrinos of extra-galactic origin. After successfully completing an engineering test flight in Ft. Sumner, NM two years after the prototype flight in Antarctica with the TIGER payload, the fully integrated radio interferometer was constructed. This chapter outlines the RF acquisition system, digitization scheme, and the overall functionality of the ANITA payload.

\subsection{Design Concept}

The ANITA experiment, depicted in figure 3.1, is a radio telescope designed to utilize the Akaryan effect from neutrino cascades produced in Antarctic ice. When a UHE neutrino interacts with an atom or molecule in ice, an electromagnetic particle shower develops that produces coherent radio emission at the Cherenkov angle. The resulting RF refracts at the ice-air interface producing a detectable impulsive radio signal observable by the payload $\sim 37 \mathrm{~km}$ above the surface. During the austral summer, a vortex develops in the upper stratosphere creating circumpolar winds which make ideal conditions for sending LDB-based experiments above the Antarctic continent.

Antarctica is an excellent site for utilizing the Askaryan effect due to low presence of RF background events otherwise detected in most parts of the world. The combined thermal noise of the ice at $\mathrm{T}_{\text {eff }} \sim 250 \mathrm{~K}$, with the receiver noise temperature allows triggering of impulsive events well into thermal noise levels which was recognized with the ANITA-lite experiment [14]. Furthermore, since Antarctica has regions of deep ice (1-4 


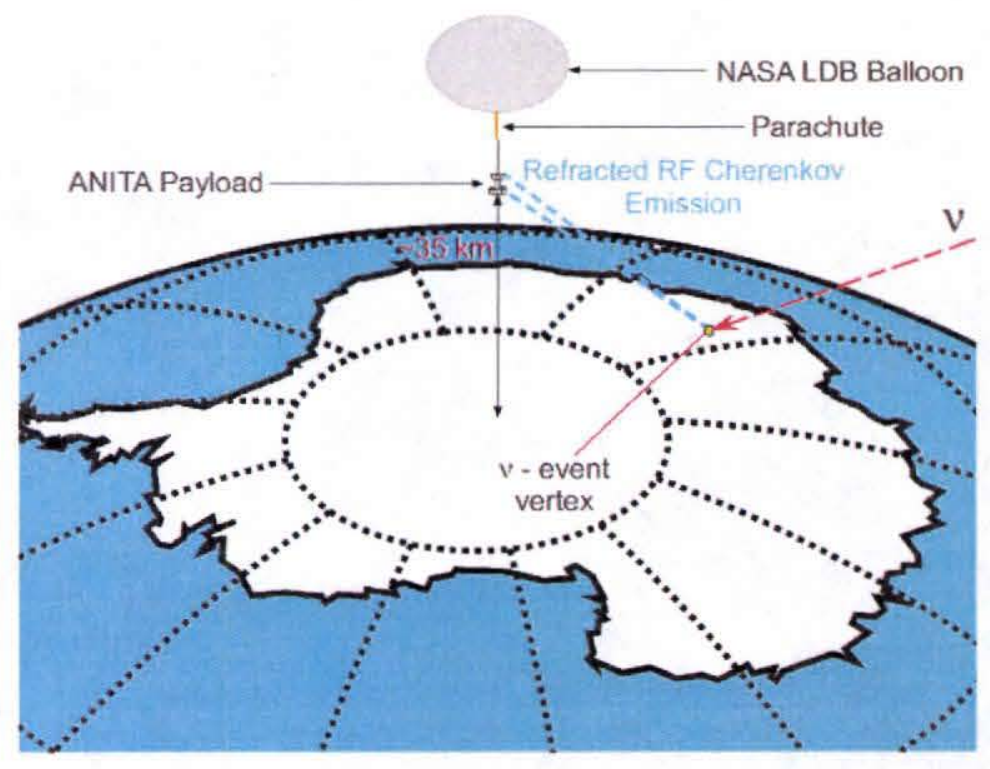

Figure 3.1: Conceptual View of the ANITA Experiment: At a float altitude of $\sim 37 \mathrm{~km}$, the ANITA payload is tethered from a NASA LDB filled with He to a volume near 2 million $\mathrm{m}^{3}$ and a diameter of $\sim 175 \mathrm{~m}$. Due to the low RF attenuation of Antarctic ice, the ANITA receiver system is able to detect EMP's emanating from $\mathrm{UHE} \nu$ 's interactions in the ice.

$\mathrm{km}$ range) that is radio transparent up to $\sim 1 \mathrm{GHz}$ [36], ANITA gains an effective telescope area of $\sim 10^{6} \mathrm{~km}^{2}$ and volumetric aperture of $1260 \mathrm{~km}^{3}$ sr at $3 \times 10^{18} \mathrm{eV}$ [37].

\subsection{Overview of ANITA Payload}

Figure 3.2 identifies the features of the ANITA payload. The bulk of the $\sim 1905 \mathrm{~kg}$ payload is composed mainly of 3 enclosures that contain the following:

1. ANITA Instrument

Data acquisition system, GPS navigation, power system, calibration pulser, discussed in section 3.3 .

2. CSBF Support Instrumentation Package (SIP)

Controls data transfer pipeline via TDRSS and Iridium antennas, balloon control (adjusting weight of payload during flight by dumping ballast).

3. Pb-acid Batteries

Used for back-up power and are a required load by the charge controller. 
The remainder of the payload contains various antenna discussed in sections 3.2.1, and 3.3. Specifics of the CSBF instrumentation will not be discussed.

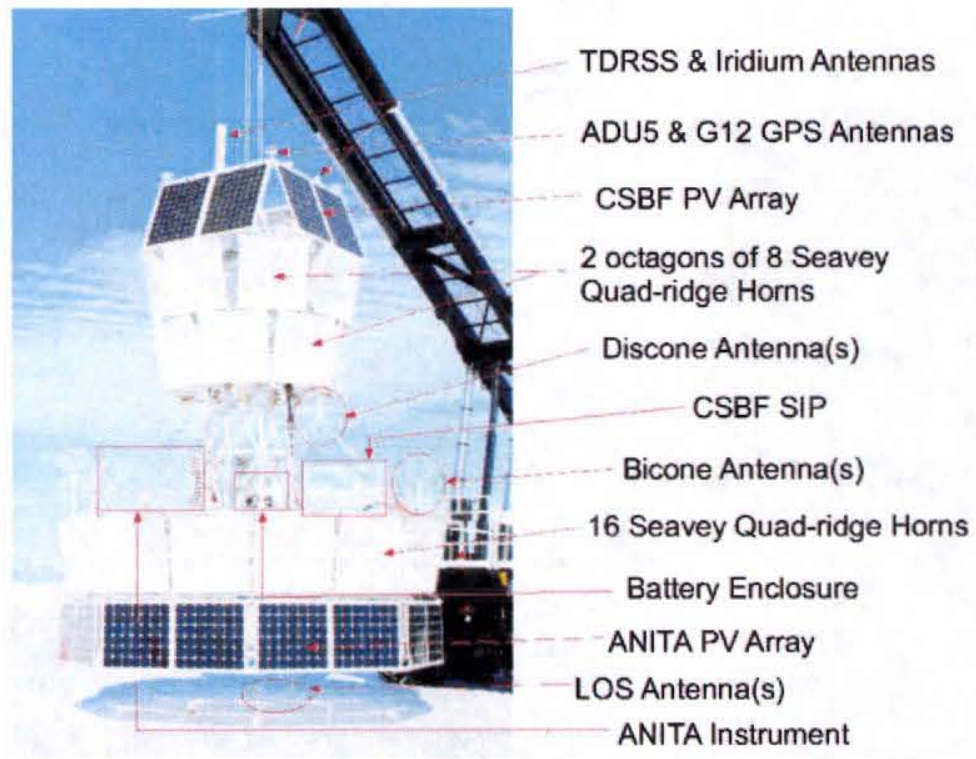

Figure 3.2: The ANITA Payload: 32 Seavey dual-polarized, quad-ridge horn antennas, 8 monitor antennas, 2 GPS systems, instrument enclosures, and 2 PV arrays for ANITA and CSBF electronics respectively.

\subsubsection{Antenna}

There are 3 types of antennas used on the ANITA payload for radio detection of Askaryan pulses. The first of these are the Seavey dual-polarization (Horizontal \& Vertical), quad-ridge horn antennas which have excellent frequency and temporal response over 200-1200 MHz. These antennas are mounted with a $\sim 10^{\circ}$ tilt from the $+\mathrm{z}$-axis defined to be the vertical axis of the payload. Sixteen are mounted on the top ( 2 octagons of 8 ) and bottom ( 1 octagon of 16 ) of the gondola, totalling 32 horn antennas. Figure 3.3 illustrates the front-end RF receiver system.

The second and third group of antennas are custom designed bicone and discone's. These serve as monitor antennas that compliment the 32 horn antennas due to their vertically polarized broadband frequency response. There are 4 antennas in each group which are mounted at deck level of the gondola. The bicones also serve as calibration pulsing antennas since they are capable of both transmitting and receiving RF signals. 


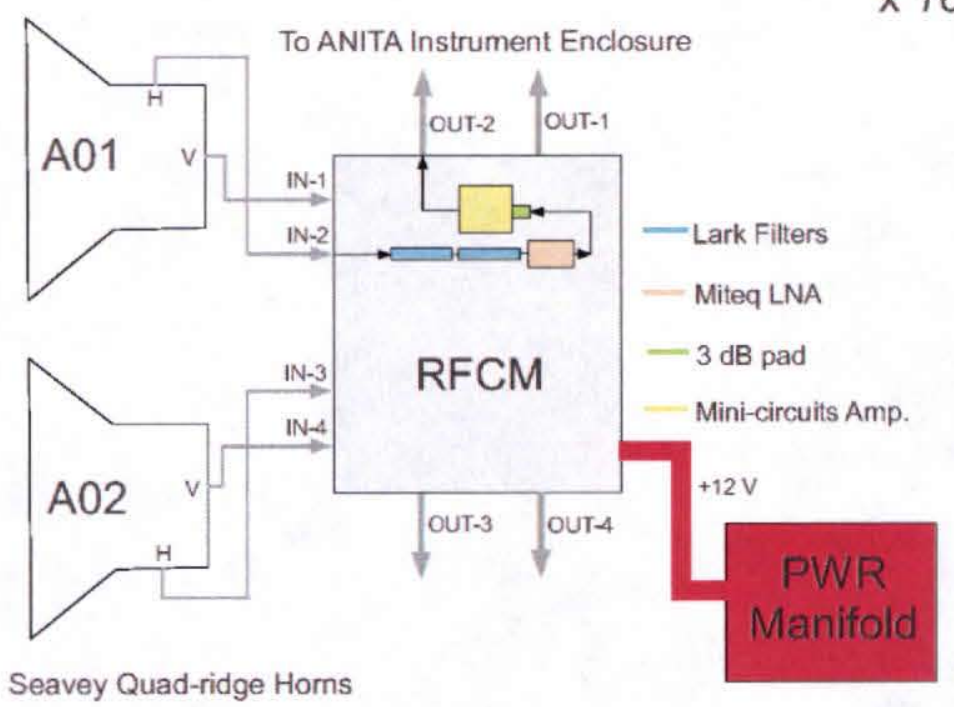

Figure 3.3: RF Receiver System: Schematic view of the front end RF system. Incoming radio waves are captured with Seavey quad-ridge horns. Signals from 2 antennas are fed through an RFCM where the signal is bandpass filtered and amplified by $80 \mathrm{~dB}(40 \mathrm{~dB}+$ $40 \mathrm{~dB}$ ). The outgoing signal is then sent to the ANITA instrument.

\subsubsection{Gondola}

The ANITA gondola was designed for quick recovery using the twin-otter recovery plane in Antarctica. The skeleton on the gondola was built with aluminum tubing, chords, radials, and trusses. Most of the mechanical assembly is done with drift pins, and 1/4-turn fasteners with a small compliment of nuts, bolts and tools. Typically, a topdown approach is used during assembly, constructing the tier 1-2 and tier 2-3 levels on the ground first. The additional column tiers (3-4 \& 4-5) and deck level (tier 5-6) are added afterwards while the payload is suspended on a crane hoist.

Once assembled, the payload is divided into $16 \phi$-sectors to identify the pointing direction of each antenna [38]. This is noted in table 3.1. An explanation for this configuration is discussed in section 3.3.2. 
Table 3.1: $\phi$-sector assignments for the ANITA payload

\begin{tabular}{|c|c|c|c|c|c|}
\hline$\phi$-sector & Top Ant. \# & Bottom Ant. \# \\
\hline \hline 1 & A08, A09, A01 & A32, A17, A18 & \multicolumn{1}{|c}{$\phi$-sector } & Top Ant. \# & Bottom Ant. \# \\
\hline 2 & A09, A01, A10 & A17, A18, A19 & A04, A13, A05 & A24, A25, A26 \\
10 & A13, A05, A14 & A25, A26, A27 \\
3 & A01, A10, A02 & A18, A19, A20 & 11 & A05, A14, A06 & A26, A27, A28 \\
4 & A10, A02, A11 & A19, A20, A21 & 12 & A14, A06, A15 & A27, A28, A29 \\
5 & A02, A11, A03 & A20, A21, A22 & 13 & A06, A15, A07 & A28, A29, A30 \\
6 & A11, A03, A12 & A21, A22, A23 & 14 & A15, A07, A16 & A29, A30, A31 \\
7 & A03, A12, A04 & A22, A23, A24 & 15 & A07, A16, A08 & A30, A31, A32 \\
8 & A12, A04, A13 & A23, A24, A25 \\
16 & A16, A08, A09 & A31, A32, A17 \\
\hline
\end{tabular}

\subsection{Data Acquisition System, Subsystems and Integrated Hard- ware}

Once an RF signal is fed through a quad-ridged horn, the two polarizations are guided into an RFCM (RF Conditioning Module) where the signal is bandpass filtered and amplified with a Miteq LNA with $\mathbf{4 0} \mathrm{dB}$ of gain. A second stage amplifier (Mini-Circuits) adds another $40 \mathrm{~dB}$ of gain to the outgoing signal that is sent to the instrument enclosure for digitization. This section outlines ANITA's electronics system.

\subsubsection{Power Consumption}

Without the use of a rotator to keep solar panels positioned toward the Sun, and omni-directional photovoltaic array is necessary. ANITA uses $8 \mathrm{PV}$ panels with 84 cells per panel located at the bottom of the payload seen in figure 3.2. The total power consumption while in flight ranges from $600 \mathrm{~W}$ at McMurdo, Antarctica latitudes up to $\sim 800 \mathrm{~W}$ at $71^{\circ} \mathrm{S}$ latitude [39].

Sixteen lead-acid batteries are also flown in case of $\mathrm{PV}$ failure and are required by the charge controller. The battery enclosure, located at deck level in figure 3.2, also serves as a counter balance for weight distribution between the ANITA instrument enclosure and the SIP enclosure.

\subsubsection{SURF, TURF, and TURFIO}

From the RFCM, the signal is carried from the upper antennas over $3.76 \mathrm{~m}$ of Andrew heliax with $50 \Omega \mathrm{N}$-type termination into instrument enclosure. The upper cable 
length differs from the bottom cable length $(4.85 \mathrm{~m})$ to ensure that received pulses will arrive at the data acquisition boards within the trigger time windows. Inside the instrument enclosure, the signal is bandpass filtered and carried over $\sim 7 \mathrm{ft}$ of $50 \Omega$ SMA terminated RG232 cable to hybrid filters that convert the horizontal and vertically polarized signals to LCP and RCP signals. Each polarization is then digitally split into 4 discretely filtered frequency bands for triggering (200-400 MHz, 400-600 MHz, 600-800 MHz, \& 800-1200 $\mathrm{MHz}$ ) by the SHORT (SURF High Occupancy RF Trigger) board and sent to the 20-slot conduction cooled, cPCI crate over $\sim 13 \mathrm{ft}$ of $50 \Omega$ SMA terminated RG232 cable.

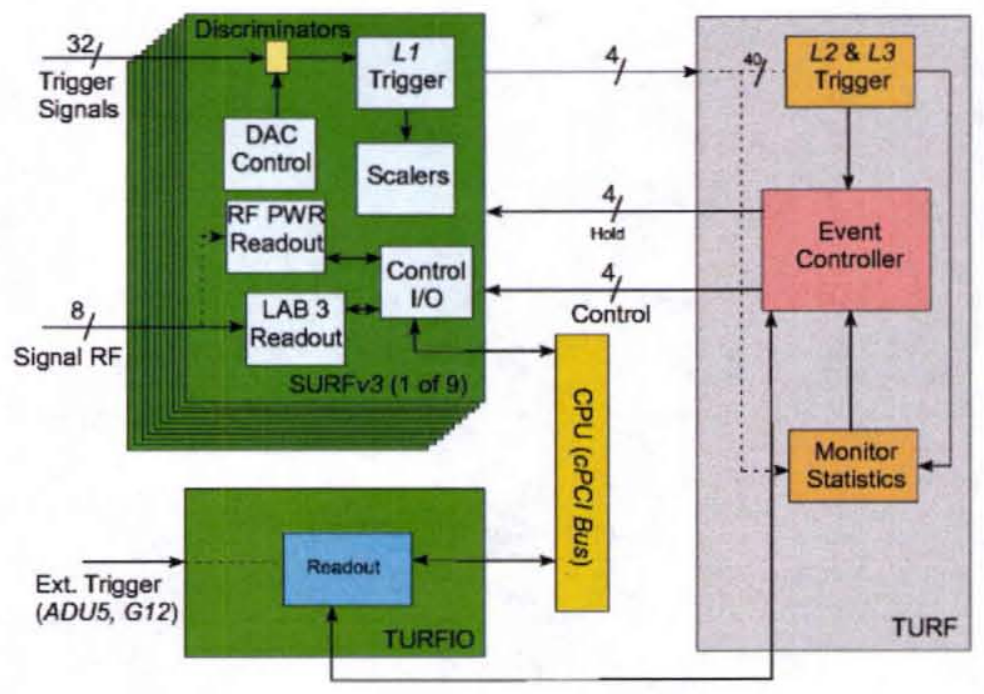

Figure 3.4: Firmware Flow Diagram: Control logic for ANITA's data acquisition system.

The Sampling Unit for Radio Frequencies (SURF) [1] forms a level-1 (L1) trigger in firmware characterized by 3 of 8 trigger bits being above threshold. The L1 trigger is then sent to the Trigger Unit for Radio Frequencies (TURF) where the event controller forms a level-2 (L2) trigger when 2-of-3 antennas are hit in the same $\phi$-sector in either the top or bottom antenna cluster. If there is a coincidence L2 trigger formed for both the top and bottom antenna cluster in the same $\phi$-sector, the TURF event controller issues a global level-3 (L3) trigger. The TURF board also serves to issue hold, and digitize signals to begin SURF ADC conversion while also monitoring trigger rates. Meanwhile, the Trigger Unit for Radio Frequencies Input and Output (TURFIO), receives an external 1 PPS trigger from the GPS units ( 3.3.3) and allows for precision timing of events by the event controller. Figure 3.4 illustrates the functionality of the ANITA firmware for data acquisition. 
Table 3.2: Triggering and sampling capabilities of the ANITA experiment [1].

\begin{tabular}{|c|c|c|}
\hline Parameter & Quantity & Comments \\
\hline \hline \# of RF Channels & 72 & 32 Top; 32 Bottom; 8 VETO \\
Sampling Rate & $2.6 \mathrm{GSa} / \mathrm{s}$ & > Nyquist \\
Sampling Resolution & $\geq 9 \mathrm{bits}$ & 3 bits noise + dynamic range \\
Samples per window & 260 & $100 \mathrm{~ns}$ window \\
\# of Sample buffers & 4 & Allows multi-hit \\
Power per Channel & $<1 \mathrm{~W}$ & Excluding LNA and triggering \\
\hline \# of Trigger Bands & 4 & $0.2-0.4 ; 0.4-0.65 ; 0.65-0.88 ; 0.88-1.2 \mathrm{GHz}$ \\
\# of Trigger Channels & 8 & Per Antenna (4 bands from RCP \& LCP) \\
Trigger Threshold & $\leq 2.3 \sigma$ & Operation into thermal noise \\
Accidental Trigger Rate & $\leq 5 \mathrm{~Hz}$ & DAQ/processing limit \\
L3 Coincidence window & $\sim 20 \mathrm{~ns}$ & Relative delay between upper and lower L2 \\
\hline
\end{tabular}

The SURF board digitization relies on the LABRADOR ASIC design [40] which has gone through three iterations to achieve the desired sensitivity for ANITA. With $1 \mathrm{GHz}$ of analog bandwidth, the LAB3 stores 9 channels of 260 deep sampling while supporting 12-bit digitization and readout in under $50 \mu \mathrm{s}$. Table 3.2 summarizes the design properties of the ANITA trigger and digitizer system.

\subsubsection{GPS}

Two GPS units are flown on the ANITA payload:

\section{Thales Navigation $G 12$}

The G12 unit provides precision horizontal positioning accuracy of $90.0 \mathrm{~cm}$ and 160.0 $\mathrm{cm}$ in vertical position using [41]. The PPS output on this until passes through a TTL boost module which forms a trigger on the TURFIO to increase trigger capability from ground pulser events.

\section{Thales Navigation ADU5}

The ADU5 unit provides differential processing of attitude, position, velocity, and time data using 4 independent receivers [42]. Further monitoring values such as heading, roll, and pitch can easily be derived from its output. The PPS output, similarly, passes through a TTL module creating a $1 \mathrm{~Hz}$ trigger used to synchronize and calibrate the TURF event time encoding. The ADU5 PPS is also used to trigger the Aotech calibration pulser. 
It should also be mentioned that two separate TTL boost units are used to drive the outputs from the GPS units to $\sim 3$ V. Both signals are sent from the TTL boost over $50 \Omega$ co-axial cable to the TURFIO and Avtech external trigger inputs where they are terminated again at $50 \Omega$.

\subsubsection{Calibration Pulser}

The Avtech pulser provides the capability to calibrate the response of the Seavey quad-ridge hom antennas while in flight. Operating on a TTL trigger input, pulses are sent at varying amplitudes via a 4-port RF switch and variable attenuator from the bicone antennas described in 3.2.1. The pulse rate is $\sim 1 \mathrm{~Hz}$ is transmitted in a rotating progression starting from bicone $1 \rightarrow$ bicone 4 .

\subsubsection{External Sensors}

Several sensors are also flown on the payload to further positioning capabilities. These include:

\section{Sun Sensors}

2-axis sensor designed to measure the position of the sun with high precision. Provides alternate heading and orientation data to compliment the differential GPS system.

\section{Attitude Sensors (accelerometers, magnetometer)}

3-axis magnetometer is used in to determine orientation in case of GPS failure by measuring the Earth's magnetic field in 3 directions. The 2 flown accelerometers compliment the magnetometer in case of GPS blackout and provide precision orientation data.

\section{Temperature Sensors}

Used to monitor the effective temperature of electronics to ensure operation within temperature limits. Typical range during balloon flights is -60 to $+100^{\circ} \mathrm{C}$. For ANITA, solar panels reached temperatures exceeding $90^{\circ} \mathrm{C}$ while electronics remained within the limits of the manufacturer. 


\section{Pressure Sensor}

Used to monitor and measure the internal pressure of the ANITA instrument enclosure and can also provide altitude information during flight. 


\section{Chapter 4}

\section{Electron Beam Experiment at the Stanford Linear Accelerator}

From June 19-24, 2006, the experiment, SLAC T486, was performed in the End Station A facility at the Stanford Linear Accelerator Center to measure the Askaryan effect in ice. $28.5 \mathrm{GeV}$ electrons were accelerated with typically $10^{9}$ particles in 10 picosecond bunches and delivered into a 7.5 metric tonne target of carving-grade ice to produce electromagnetic showers. In a dense media like ice, coherent microwave Cherenkov radiation emerges from the particle shower and propagates to the surface of the target where radio anternas can detect the radiation. This chapter outlines the T486 experiment and the analysis of the Askaryan effect in ice.

\subsection{Experimental Setup}

Figure 4.1 shows the overall setup of T486 at ESA. 28.5 GeV electrons were accelerated with the SLAC Linac and deposited into a $\sim 7.2$ metric tonne ice target ( $n_{\text {ice }}=1.8$ ). From the Askaryan process, Cherenkov radiation propagates out of the target at an angle of $\sim 56^{\circ}$ where it refracts at the surface carved to a mean slope of $\sim 8^{\circ}$. The resulting emission was then detected with the ANITA receiver system at an angle of $\sim 25^{\circ}$ from the electron beam axis. In addition to the ANITA payload, several exterior antennas (LPDA, Standard Gain Horn, Dipole, etc.) were placed around the ice target and are not shown in figure 4.1 but will be discussed in section 4.1 .2 and will be the emphasis of this analysis. 


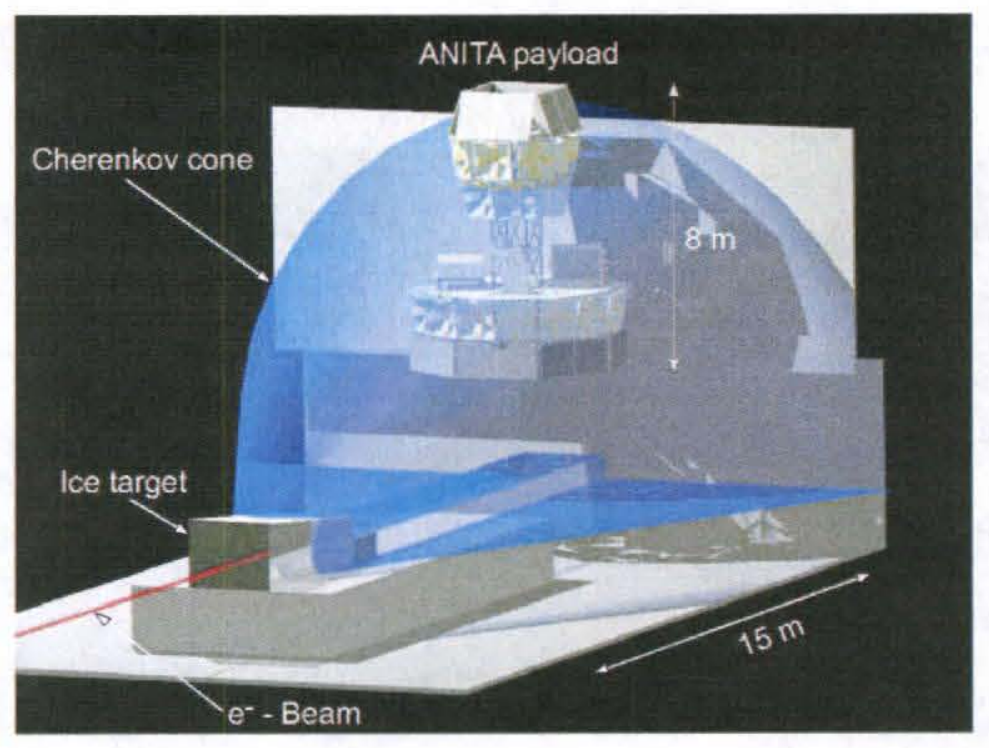

Figure 4.1: SLAC T486 Experimental Setup: Rendered view of the ANITA payload with the $28.5 \mathrm{GeV}$ electron beam at SLAC's ESA.

\subsubsection{Beam Current}

The electromagnetic showers were produced with $28.5 \mathrm{GeV}$ electrons accelerated by the SLAC Linac. In most cases, $10^{9}$ particles were delivered in 10 picosecond bunches. Two toroids within the Linac were used to monitor the beam current. Toroid 34 is located upstream in the beam switchyard and seems to be somewhat unreliable at low currents due to the granularity of it's ADC. Further downstream, toroid 4140 was set up after the experiment started as seen in figure 4.2 and has an overall gain correction factor of 10 in order to correlate the 2 sets of data. For the purpose of matching beam current with antenna peak voltages, only toroid 34 will be used in the analysis.

\subsubsection{RF Monitor and External Antennas}

Several exterior antennas were setup as additional monitors from the ANITA payload for SLAC T486. A dipole antenna, depicted in figure 4.3, served as an absolute beam current monitor and as a calibration for the remainder of the antennas receiving Askaryan pulses emanating from the ice. Two LPDA model EM-6952 antennas manufactured by Electro-Metrics with 1-18 GHz of bandwidth were mounted on a wooden structure positioned on a concrete wall that stood $\sim 5 \mathrm{~m}$ from the center of the ice target. One LPDA was 


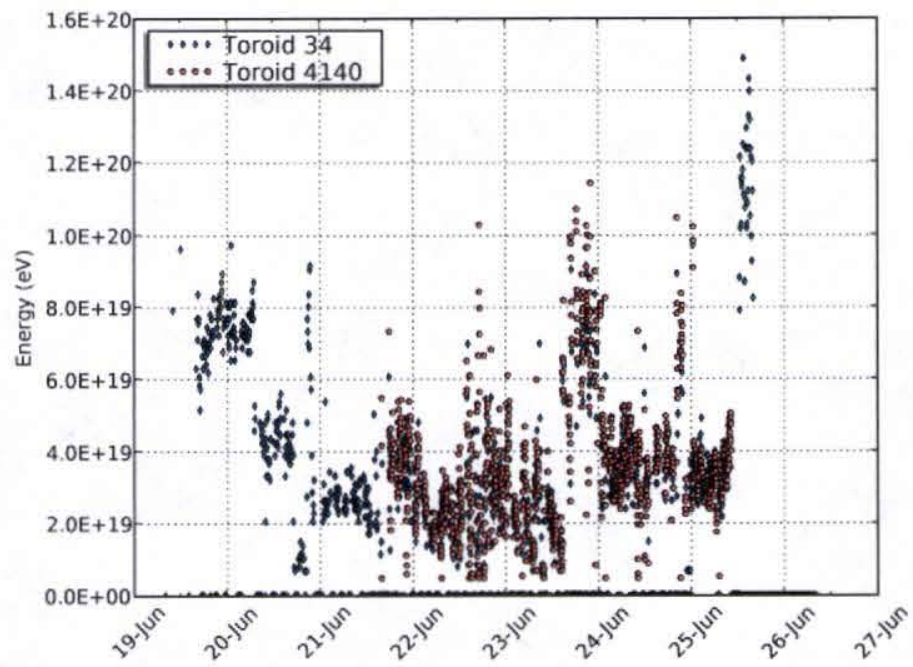

Figure 4.2: SLAC T486 Beam Current: Beam Current vs. time showing the number of electron bunches per unit energy per unit time. The data sets came from 2 individual monitoring toroids which span the duration of the experiment.

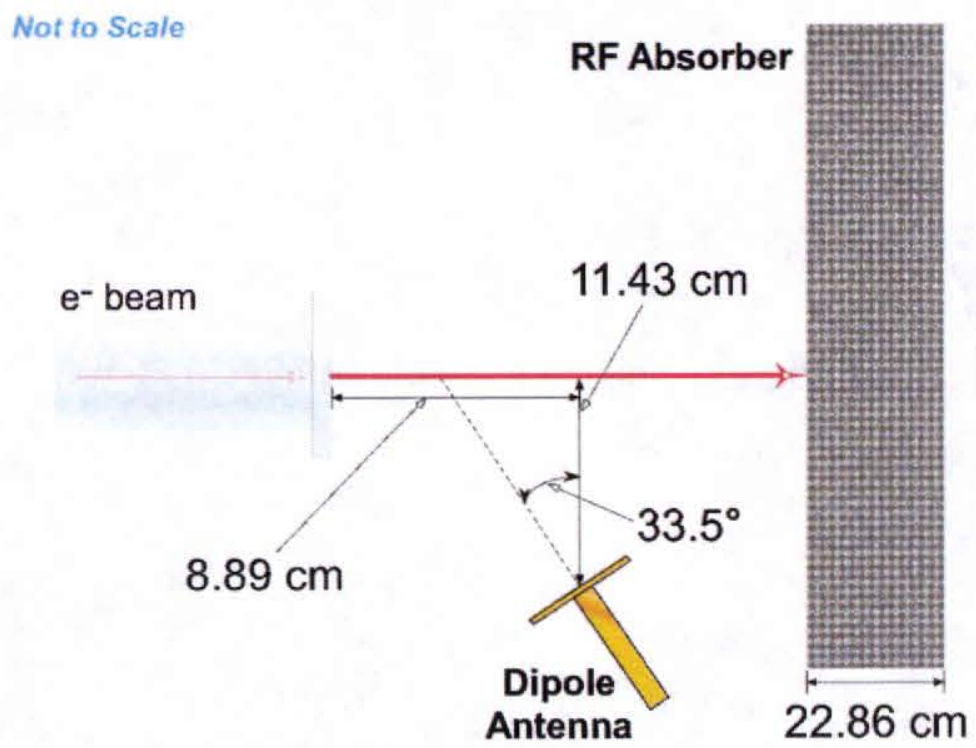

Figure 4.3: Diagram showing the relative position of the dipole antenna to the beam pipe and the external RF absorber. 
directed in the horizontal plane while the other in the vertical plane. Two custom-made Monocone antennas were positioned on a similar wooden structure on a concrete wall adjacent to the LPDA's on the other surface. Each monocone was directed in the horizontal and vertical plane $\sim 5 \mathrm{~m}$ from the center of the ice target which stood between the two concrete walls. Below the two monocone's, a Scientific-Atlanta Standard Gain Horn model 12-2.6 with 2.6-3.95 GHz of bandwidth was positioned $\sim 4.5 \mathrm{~m}$ from the center of the ice and aligned with boresight. In addition to the RF antennas mentioned, two PCB LPDA's were placed below the LPDA's mentioned previously but will not mentioned in the data analysis. The description of the overall antenna setup is shown in figure 4.4

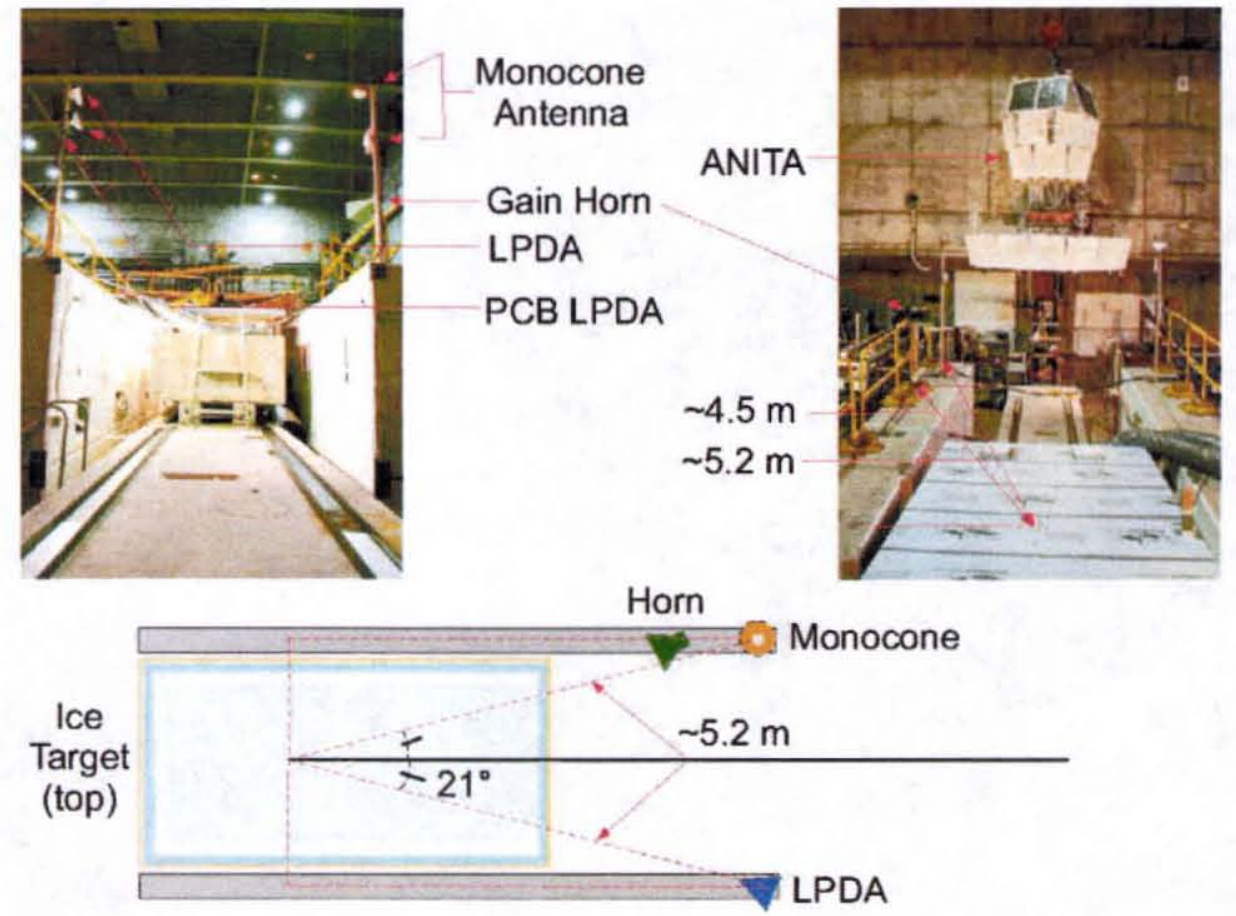

Figure 4.4: Antenna positions for T486: Top left and right show the positions of the gain horn, LPDA's, Monocones, PCB LPDA's, and the ANITA instrument relative to the ice target.

\subsubsection{Ice Target}

The ice target for T486 was constructed using $~ 55136 \mathrm{~kg}$ blocks of carving grade ice. Each block, with dimensions $25.4 \mathrm{~cm} \times 53.3 \mathrm{~cm} \times 114.3 \mathrm{~cm}$, was lowered into the target housing made predominately of $1.9 \mathrm{~cm}$ plywood to form a monolithic block $2 \mathrm{~m} \times 1.5 \mathrm{~m}$ 
$\times 5 \mathrm{~m}$. The housing was lined with $10.2 \mathrm{~cm}$ foam thermal insulation with a thin layer of hypalon liner to contain any melting water. Beneath the ice block and hypalon liner was a layer of $10 \mathrm{~cm}$ ferrite tile to suppress reflections of Cherenkov radiation that was generated downwards. The entire volume was regulated between $-5^{\circ} \mathrm{C}$ to $-20^{\circ} \mathrm{C}$ throughout the duration of the experiment.

The top surface of the ice target was carved to a slope of $\sim 8^{\circ}$ to prevent total internal reflection (TIR) from Cherenkov emission near the surface. The roughness of the surface after carving was measured to have a $2.3 \mathrm{~cm}$ root-mean-square (rms). Figure 4.5 shows some photographs of the ice surface and the target housing with the ice surface grid-map superimposed in the bottom-left photograph.
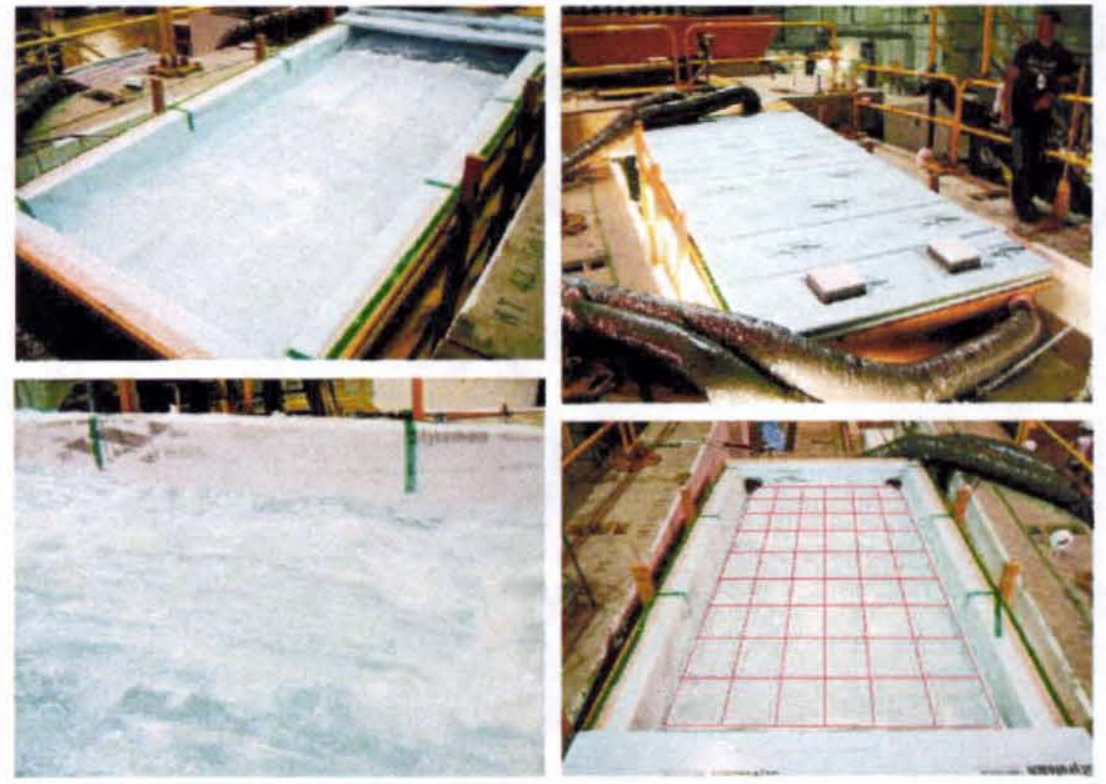

Figure 4.5: Photographs of SLAC T486 Ice Target: (Top left) Front view of ice target with $\sim 8^{\circ}$ slope. (Top right) Front view of ice target with foam insulation cover and cooling ducts shown. This was the configuration for the majority of runs during the experiment. (Bottom left) Close-up showing the surface roughness of the ice target. (Bottom right) Top view of ice target with measured surface roughness grid from appendix $A$ (not to scale).

Surface Roughness Several measurements were made on the completed surface to quantify effects of surface roughness that will have more importance with data analysis from the December 2006 ANITA flight. A $13 \times 6$ element grid was developed to get a precise value of effective surface height and slope. At each grid point, an effective surface height 
and slope were measured and summarized in appendix A. Figure 4.6 shows the design of the ice target and the layout of the constructed surface grid and the measured surface is plotted in figure 4.7 .

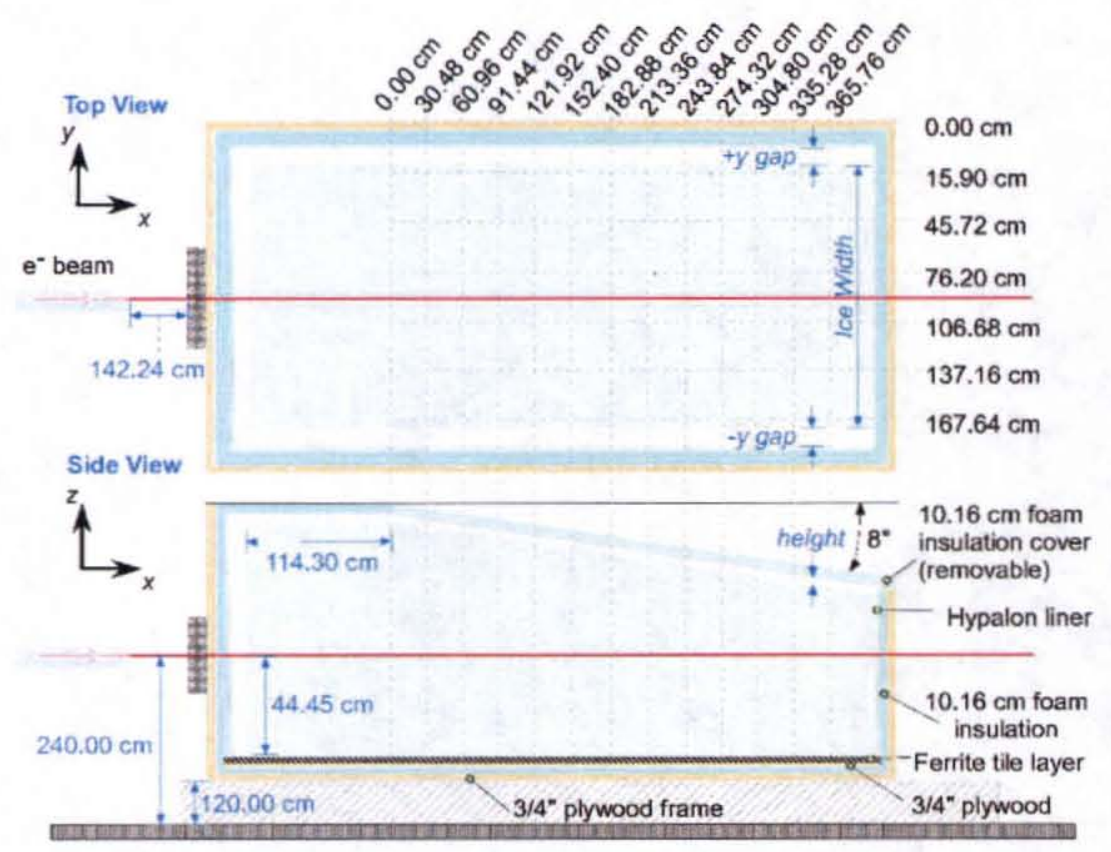

Figure 4.6: Schematic view of the Ice Target's Surface Roughness: The above describes the physical layout of the ice target for T486 (not to scale).

Due to the apparent change in slope of the ice target surface, it becomes necessary to divide the ice surface into two distinct regions - A \& B. Figure 4.8 shows the division of the ice surface into these regions. By fitting a plane to each surface ( $h_{f i t}=A \times l+B \times w+C$ ), which is shown in red, to the measured grid points (blue), two slopes can be derived. For Region A, the fit reveals a slope of $8.4^{\circ}$ while Region B has a slope of $2.6^{\circ}$. Since shower max occurs in Region A from $\sim 2.4 \mathrm{~m} \rightarrow \sim 2.8 \mathrm{~m}$, a slope of $8.4^{\circ}$ will be used in this analysis for the radiated Cherenkov emission.

\subsection{Data Acquisition}

All measurements of pulsed RF emission were made in the time domain with the ANITA instrument (described in section 3.3); a Tektronix 694C real-time digital sampling oscilloscope with $3 \mathrm{GHz}$ of bandwidth and $10 \mathrm{GSa} / \mathrm{s}$, eight-bit linear digitization of four 


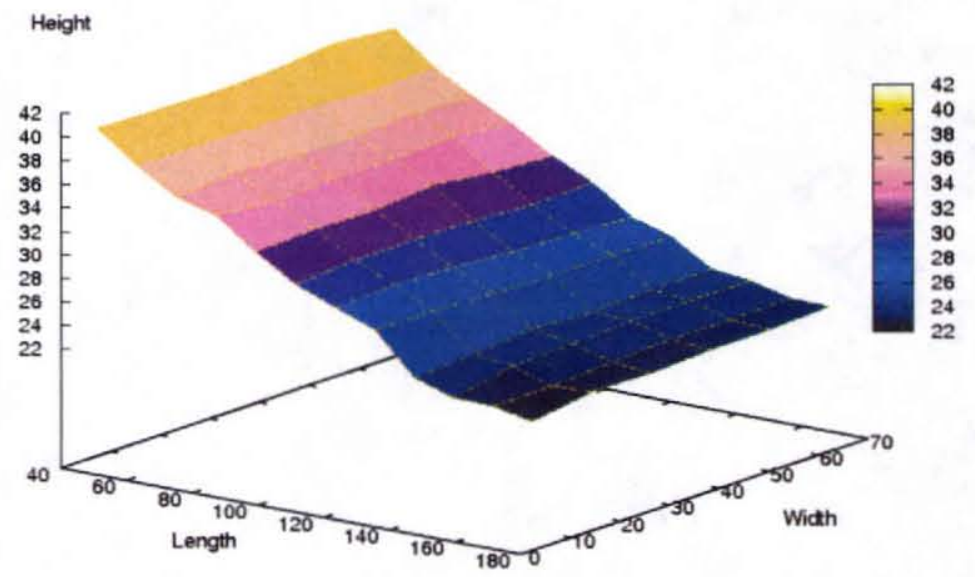

Figure 4.7: Plot of T486 ice surface. All values are in inches. Note the pronounced change in slope at $\approx 140^{\prime \prime} \approx 3.5 \mathrm{~m}$.
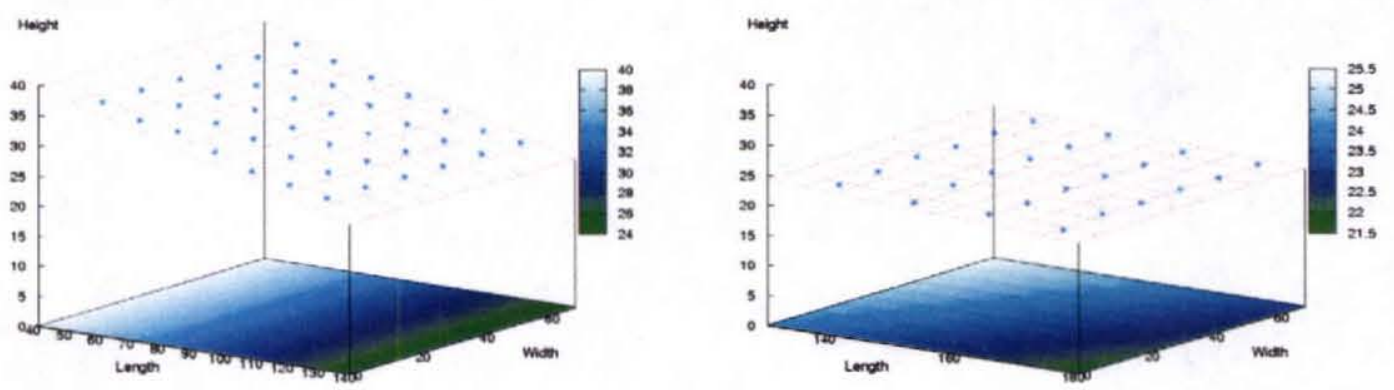

Figure 4.8: Left: Region A and Right: Region B of the T486 ice target. The fitted plane (red) to the measured grid points (blue) is shown revealing two distinct inclination angles $-8.4^{\circ}$ and $2.6^{\circ}$ respectively. All dimensions are in inches here. 
Table 4.1: A summary of the additional antenna used in T486 with their mapping to oscilloscope. The top half of the table shows the initial setup for data acquisition and the bottom half denotes the setup after 06/20/06 2am PDT.

\begin{tabular}{|l|c|c|c|c|}
\hline Antenna & Polarization & Channel & Device & Distance from Ice Target \\
\hline \hline PCB LPDA & $\mathrm{H}$ & 1 & TDS694C & $5.2 \mathrm{~m}$ \\
PCB LPDA & $\mathrm{V}$ & 2 & TDS694C & $5.2 \mathrm{~m}$ \\
Gain Horn & $\mathrm{V}$ & 3 & TDS694C & $4.5 \mathrm{~m}$ \\
Dipole & & 4 & TDS694C & N/A \\
LPDA & $\mathrm{H}$ & 1 & HP54121A & $5.2 \mathrm{~m}$ \\
LPDA & $\mathrm{V}$ & 2 & HP54121A & $5.2 \mathrm{~m}$ \\
Monocone & $\mathrm{H}$ & 3 & HP54121A & $5.2 \mathrm{~m}$ \\
Monocone & $\mathrm{V}$ & 4 & HP54121A & $5.2 \mathrm{~m}$ \\
\hline Monocone & $\mathrm{H}$ & 1 & TDS694C & $5.2 \mathrm{~m}$ \\
Monocone & $\mathrm{V}$ & 2 & TDS694C & $5.2 \mathrm{~m}$ \\
Gain Horn & $\mathrm{V}$ & 3 & TDS694C & $4.5 \mathrm{~m}$ \\
Dipole & & 4 & TDS694C & N/A \\
LPDA & $\mathrm{H}$ & 1 & HP54121A & $5.2 \mathrm{~m}$ \\
LPDA & $\mathrm{V}$ & 2 & HP54121A & $5.2 \mathrm{~m}$ \\
PCB LPDA & $\mathrm{H}$ & 3 & HP54121A & $5.2 \mathrm{~m}$ \\
PCB LPDA & $\mathrm{V}$ & 4 & HP54121A & $5.2 \mathrm{~m}$ \\
\hline
\end{tabular}

channels; and with a Hewlett-Packard 54121A Digital Mainframe Oscilloscope with 20 $\mathrm{GHz}$ of bandwidth. The signals received at the ANITA antennas were attenuated by $60 \mathrm{~dB}$ to bring signals into an acceptable range for $50 \Omega$ inputs while $20 \mathrm{~dB}$ of attenuation was used on received signals from the dipole antenna. Table 4.1 summarizes the mapping of antennas to the TDS694C and HP54121A with a switch occurring on the morning of June $20,2006$.

A typical run during T486 captured $~ 1000$ events with the TDS694C while data acquisition with the HP54121A was a "work in progress" and will not be presented in this analysis. Figure 4.9 shows a snapshot from event 888 in run 126 with an approximate shower energy of $2.3 \times 10^{19} \mathrm{eV}$.

\subsubsection{Dipole Calibration}

To calibrate the transition radiation entering the ice target, 104 runs with typically 1000-2000 events were used to build a correlation between the peak voltages received by the dipole antenna and the generated beam current. Due to the poor quality of the beam current data set, only 19 runs passed a selection criteria which was motivated by well- 

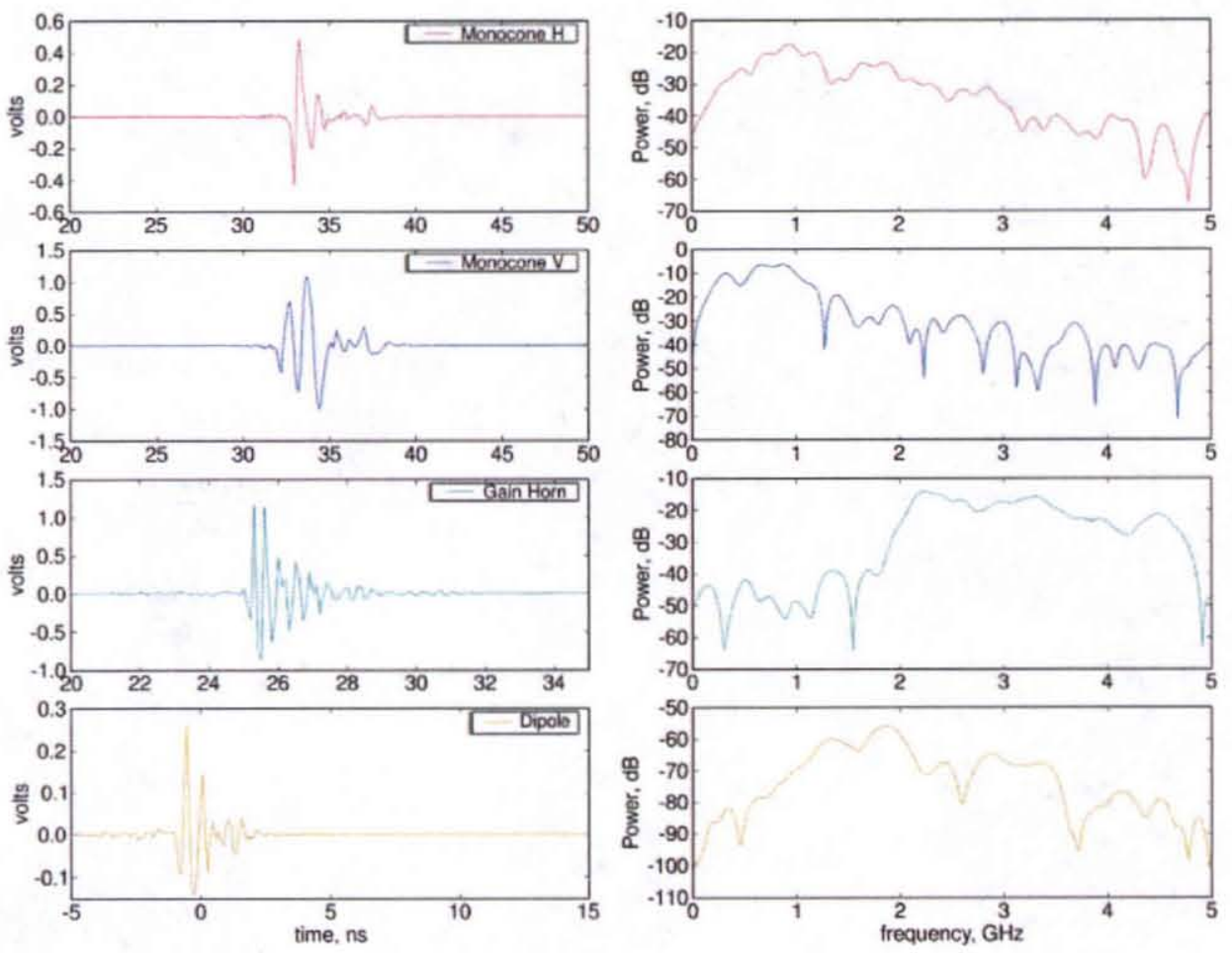

Figure 4.9: Waveforms with the respective power spectrum from run 126, event 888 . 
behaved dipole peak distributions (figure 4.10), and the beam current tracking the dipole peaks consistently over the entire run.
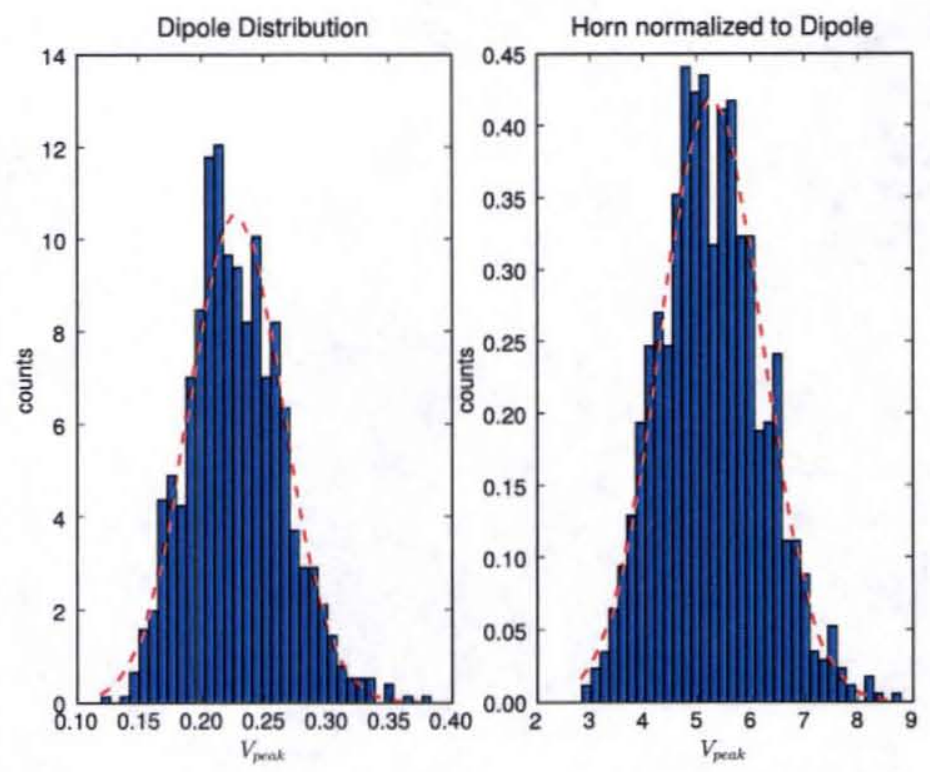

Figure 4.10: Run 102 peak voltage distributions for the dipole antenna and gain horn which is normalized to the mean of the dipole peak distribution. For run 102, the dipole distribution has $\mu=0.2273$ and $\sigma=0.0379$, which provides a normalized horn distribution with $\mu$ $=5.2602$ and $\sigma=0.9537$. All units are in relative volts.

Figure 4.11 shows the coherence of the detected electromagnetic pulse power as a function of shower energy which is directly proportional to the transition-radiation monitored beam current. This experimental result yields a slope of $2.07713 \pm 0.07846$. The quadratic dependence of the pulse power of the radiation detected in T486 provides a clear indication of coherence of Cherenkov emission.

\subsubsection{Gain Horn Calibration}

The response of the gain horn was simulated using a MATLAB routine that solves for the electric and magnetic fields from the physical dimensions of the pyramidal horn antenna. Figure 4.12 shows the $\mathbf{E}$ - and $\mathbf{H}$-plane beam patterns for the for the low, mid, and high-bands of the horn antenna.

The boresight gain response of a pyramidal horn antenna is useful for both receiving and transmitting electromagnetic signals and represents the ratio of the intensity of the 


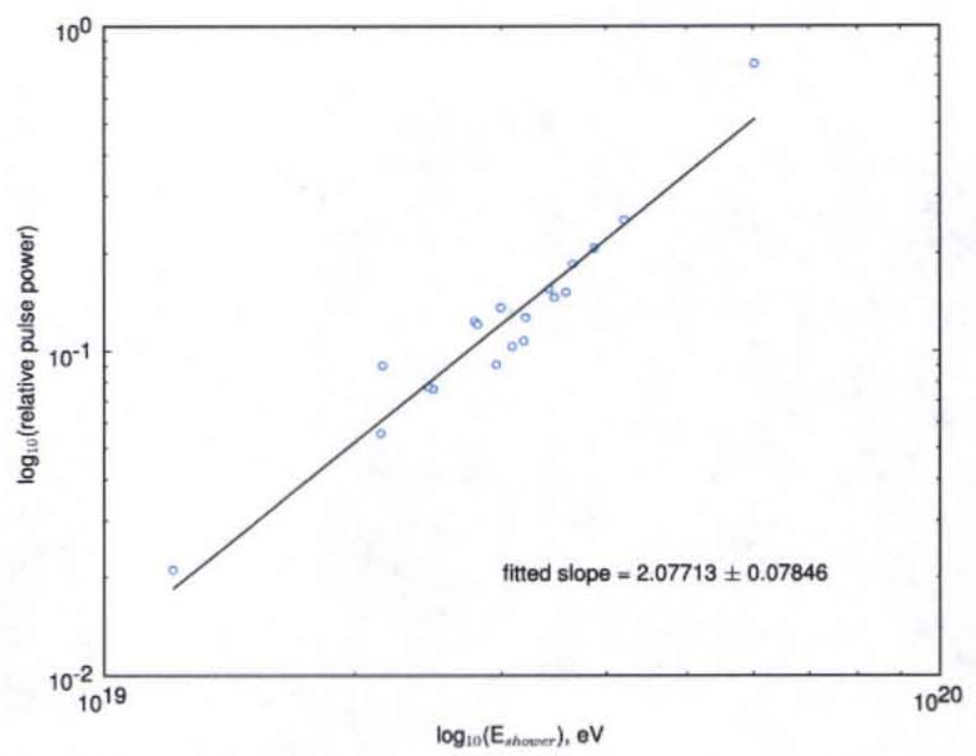

Figure 4.11: Detected pulse power as a function of shower energy for 19 runs during T486. The dipole antenna, depicted in figure 4.3 , measured the transition radiation exiting the beam pipe at an angle of $56.5^{\circ}$ from the beam axis. The quadratic rise with beam energy is characteristic of coherent radiation.

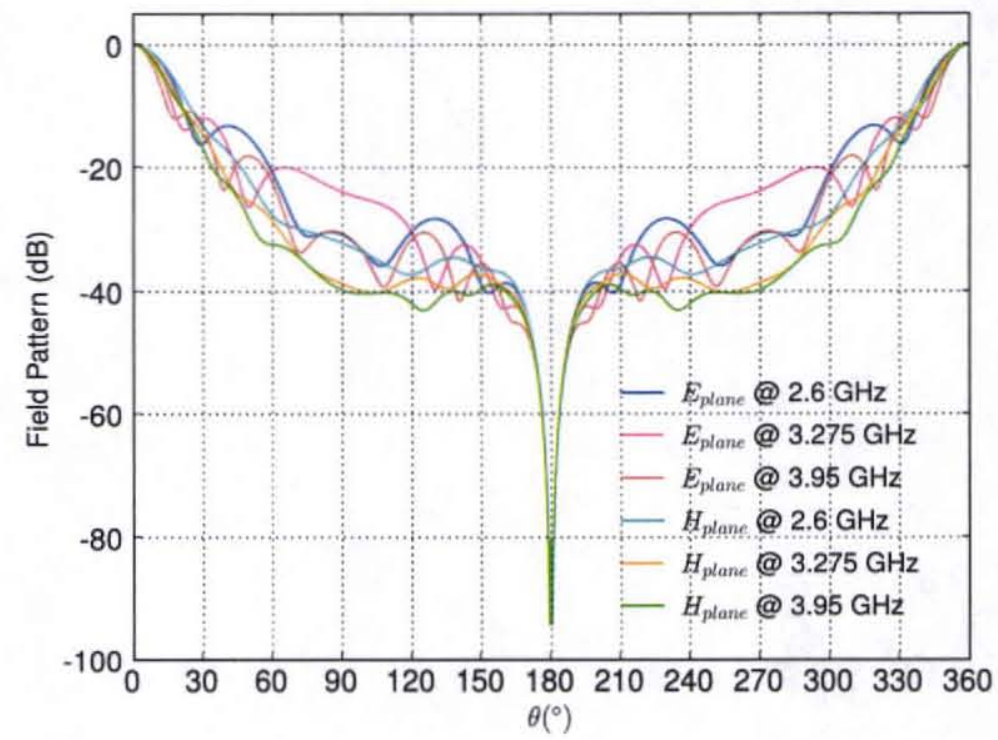

Figure 4.12: E \& H - Fields of low, mid, and high-bands for Standard Gain Horn (ScientificAtlanta, Inc.). 
antennas radiation pattern in the direction of the strongest radiation to that of a reference source. In the case of transmission, the gain determines how much RF power can be sent in the main lobe or even side lobes. This quantity is still useful to model since it is directly related to the effective area which is a measure of the area that the antenna would need to occupy to intercept observed source power. Figure 4.13 shows the results of simulating the pyramidal gain horn in both the $\mathbf{E}$ - and $\mathbf{H}$-planes.
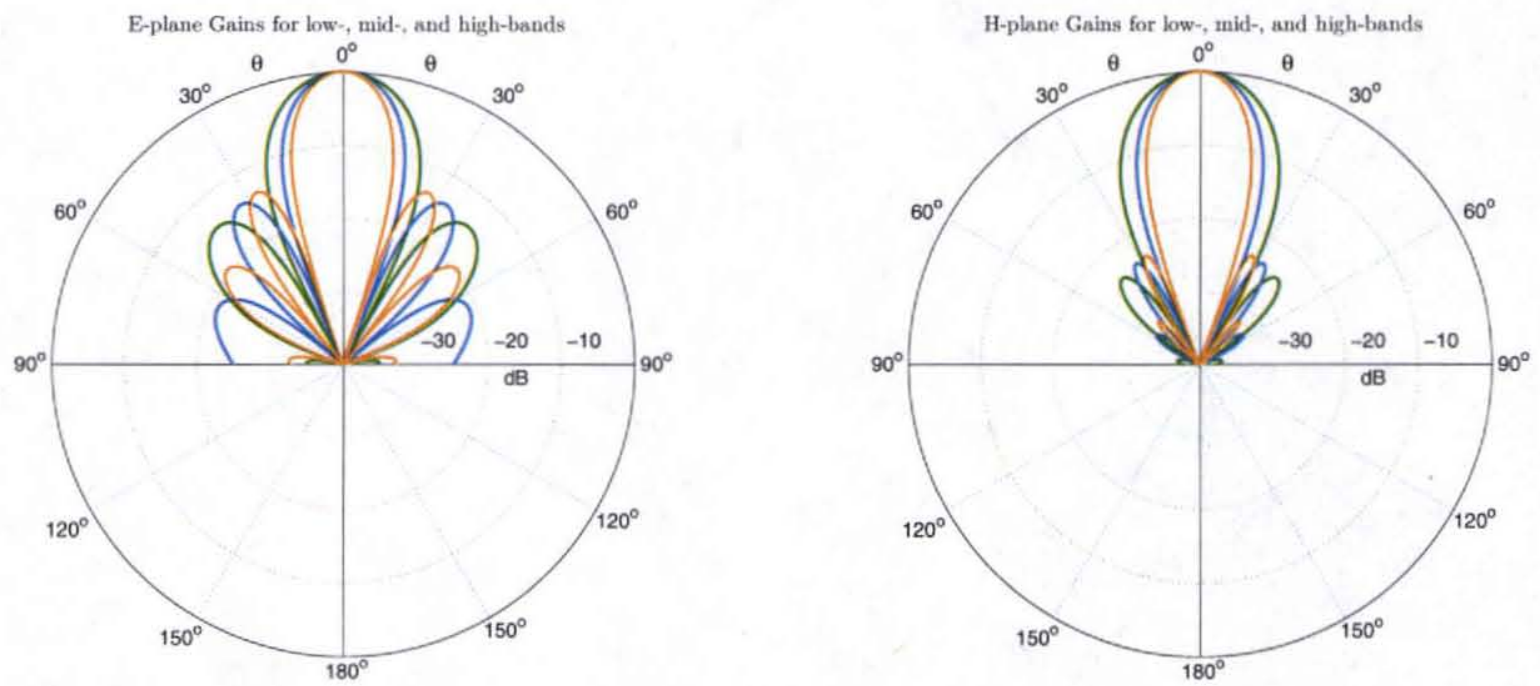

Figure 4.13: Left: E-plane gain and Right: H-plane gain for low- (green), mid- (blue), and high-bands (orange) of the horn antenna. The beam pattern narrows significantly with higher frequencies suggesting the gain should increase with frequency.

In order to calculate the effective height of the horn antenna, several parameters needed to be simulated numerically. From Krauss [43], we define the aperture efficiency as

$$
\varepsilon_{a p}=\frac{A_{e}}{A_{p}},
$$

where $A_{e}$ and $A_{p}$ are the effective area and physical area of the antenna's aperture. Since the physical area of the antenna aperture is a known quantity, we can determine the effective area with the use of another MATLAB function which determines the aperture efficiency. The effective area of an antenna is related to the boresight gain, G, and the free-space wavelength, $\lambda$, of the radiation by the formula: 


$$
G=\frac{4 \pi}{\lambda^{2}} A_{e}
$$

The directivity of the gain horn is related to the HPBW (Half-Power-Beam-Width) by

$$
D \approx \frac{4 \pi}{\Omega_{A}} \approx \frac{41253^{\circ}}{D_{H} D_{E}},
$$

where $\Omega_{A}$ is the beam solid angle, and $D_{H}$ and $D_{E}$ are the HPBW in the H- and E-plane respectively. Figure 4.14 displays the HPBW response as a function of frequency which is consistent with expectation.

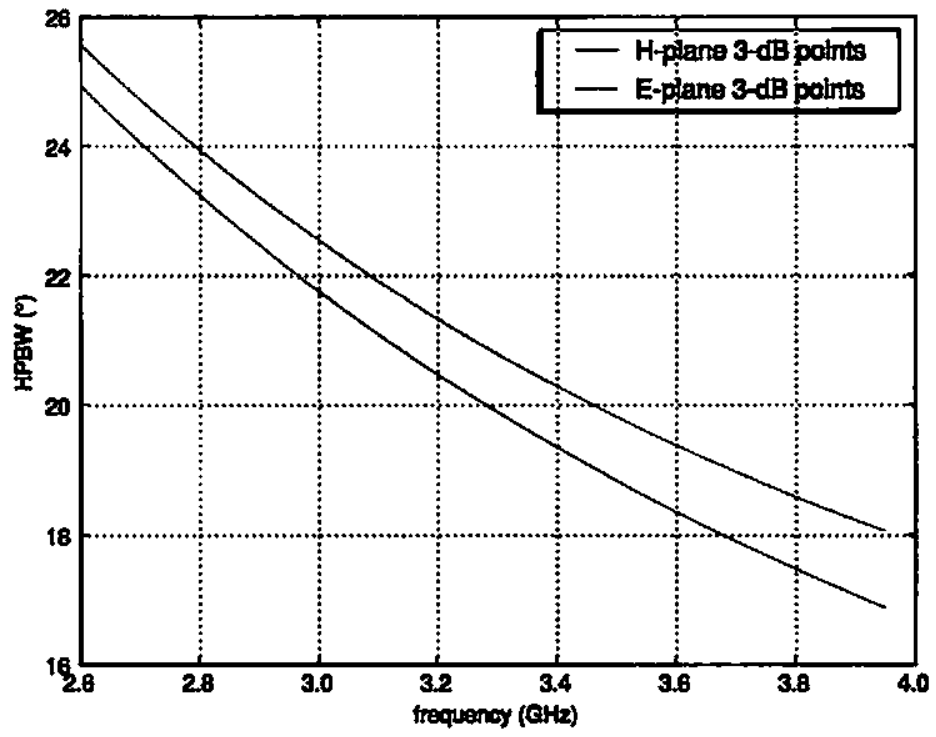

Figure 4.14: Half-power-beam-width as a function of frequency for the Standard Gain Horn showing the main lobe of the beam pattern narrowing at higher frequencies.

The results of simulating the pyramidal horn's gain and directivity are summarized in figure 4.15 showing a gradual rise in the frequency domain.

Another parameter related to the antenna aperture is the effectioe height, $h_{\text {eff }}$, which is expressed in meters. This quantity is simply the ratio of the open circuit voltage to the incident electric field:

$$
h_{e f f}=\frac{V_{o c}}{E} .
$$



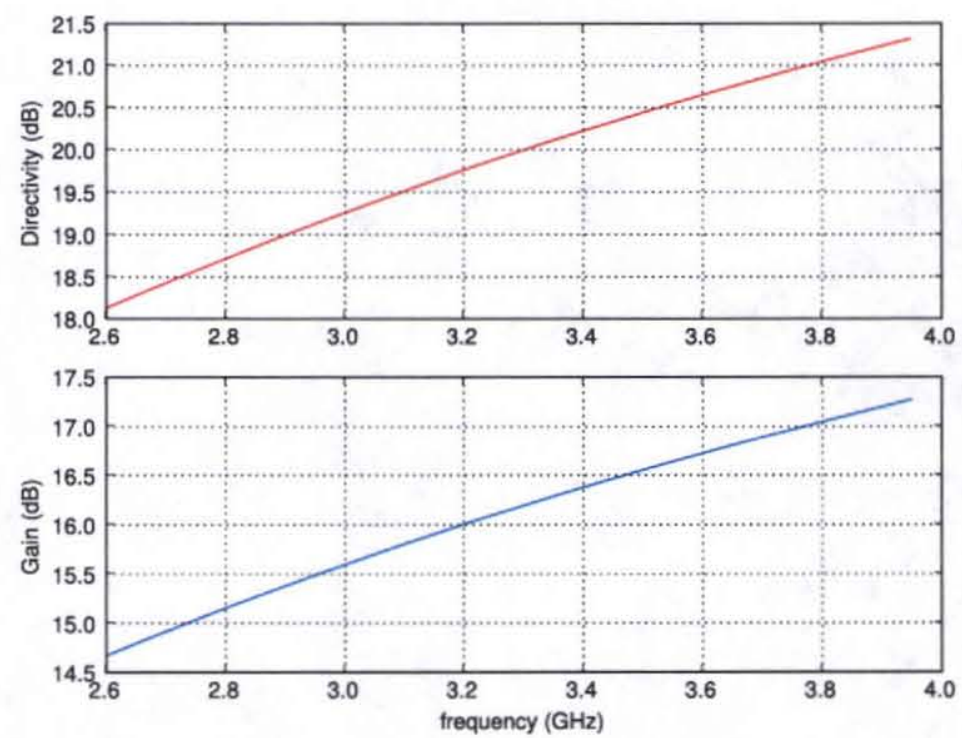

Figure 4.15: Directivity (top) and gain (bottom) as a function of frequency.

Krauss [43] expresses the effective height in terms of the effective aperture, $\mathrm{A}_{e}$, radiation resistance, $Z_{r}$, and the intrinsic impedance of free space, $Z_{0}=377 \Omega$ which is given by

$$
h_{\text {eff }}=2 \sqrt{\frac{Z_{r} A_{e}}{Z_{0}}} .
$$

Using the conditions previously outlined, the simulated results of the standard gain horn are shown in figure 4.16. The results are consistent with expectation and are modest since the effective height data contains no phase information. A better numerical simulation involves FDTD (Finite-Difference-Time-Domain) analysis [44] and is more difficult in principal. For this analysis, the effective height correction is sufficient and produces an overall scaling factor for the received field strength.

Lastly, I show the coherence of the Cherenkov emission measured by the gain horn (figure 4.17). Here a similar procedure was adopted from the dipole calibration which passed 17 runs averaging 1000-2000 events each. 

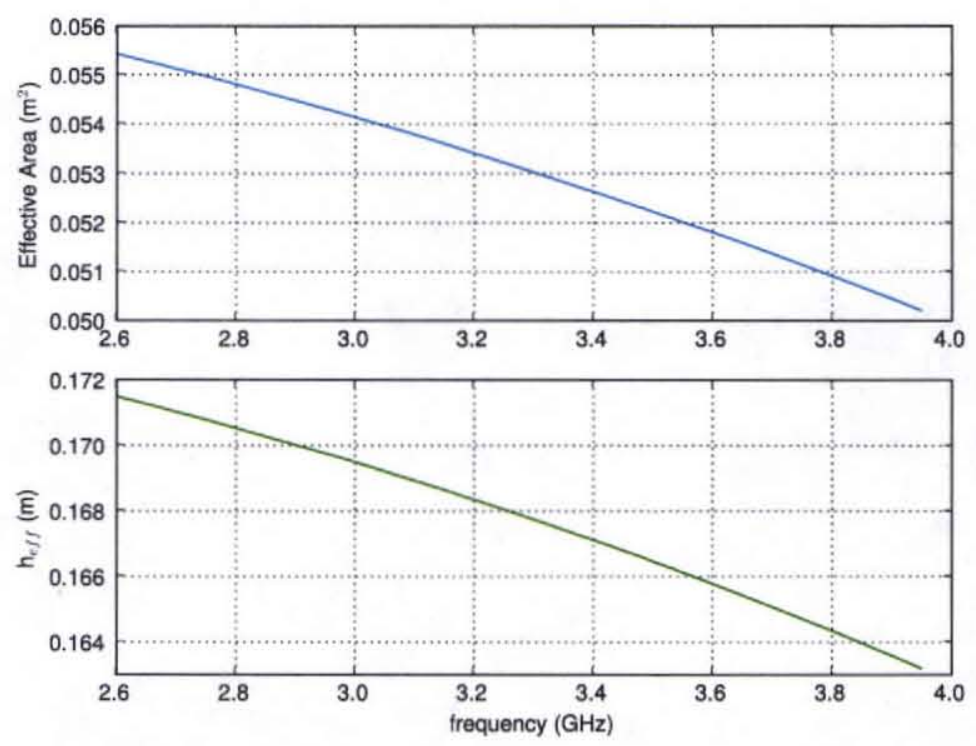

Figure 4.16: Effective area (top) and effective height (bottom) for the Standard Gain Horn as a function of frequency.

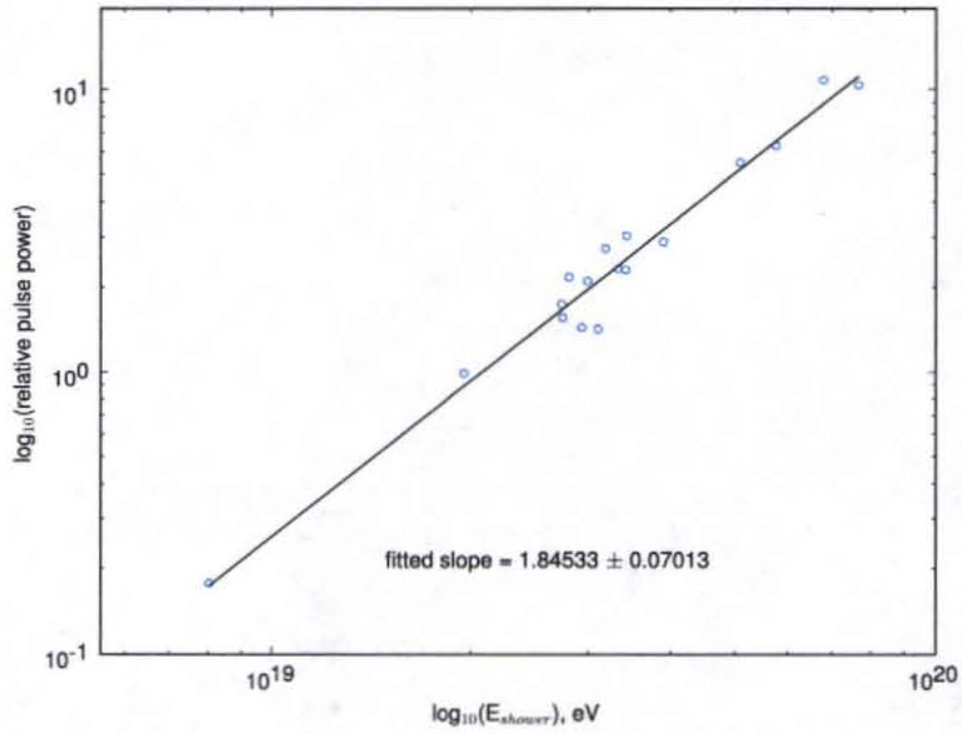

Figure 4.17: Detected pulse power as a function of shower energy for the Standard Gain Horn. 


\subsection{Electric Field Reconstruction}

The electric field of the pulsed RF Cherenkov emission can be calculated using 4.2.4. Each waveform has been zero-mean subtracted with a Gaussian window applied in the time-domain. Figure 4.18 shows a sample waveform from run 102 with the above corrections applied.
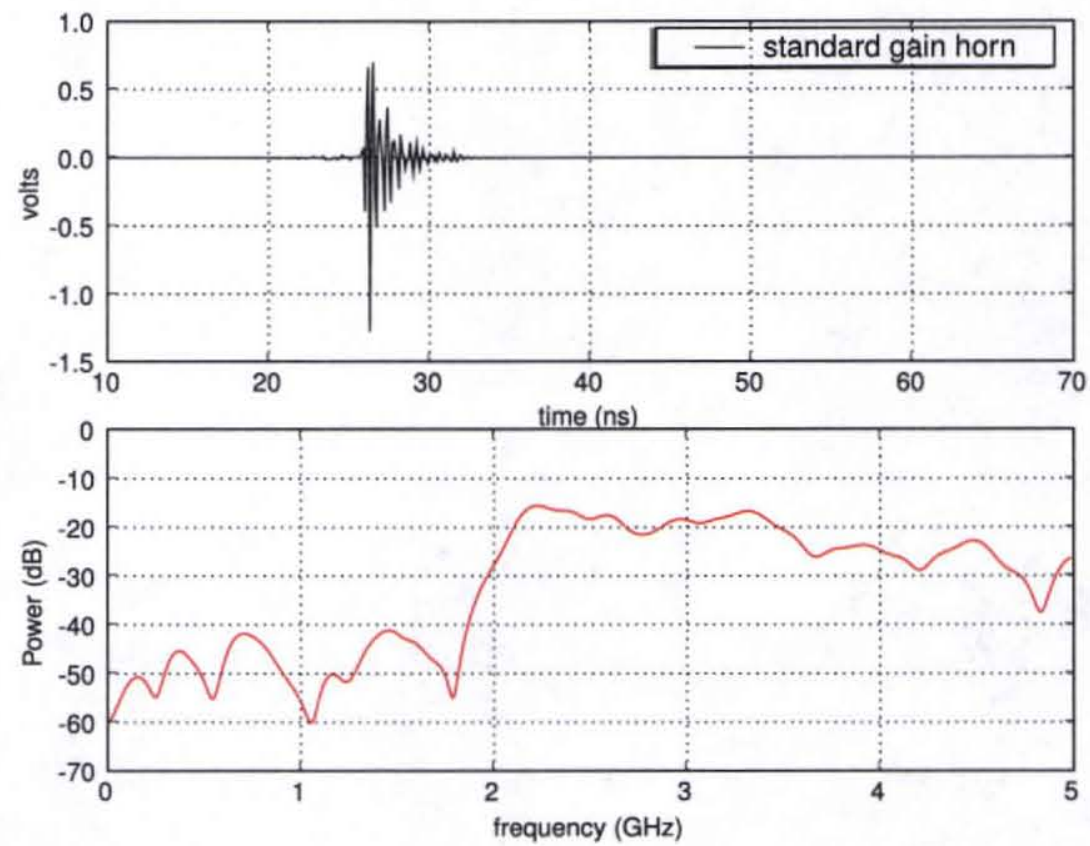

Figure 4.18: (Top) Waveform received by the horn antenna in run 102 with the corresponding power spectrum (bottom).

Effects due to cable corrections were negligible for this analysis due to the fact that the amplitude change was insignificant. Furthermore, the TDS694C only has $3 \mathrm{GHz}$ of bandwidth so a correction for the frequency roll-off of the oscilloscope is necessary. A picosecond pulse was injected into the scope and the measured frequency spectrum was recognized to have significant contributions in power above the quoted roll-off from the manufacturer. This effect, however, was not used as a correction to the data since the change in field strength was collectively unnoticeable. A time-domain representation of the effective height was not calculated, so the electric field was calculated in the Fourier-domain with 
$1 \mathrm{MHz}$ bin resolution. A careful FDTD representation of the effective height may improve the quality of these results but was not taken into account for this result.

The theory in equation 2.2 .3 was derived for a pulse detected at a large distance from a shower initiated by a single particle. In this case, we can treat the shower as a single source emitter with radiation coherent over the full length of the shower. Figure 4.19 shows the measured electric field strength for several different antennas used during T486.

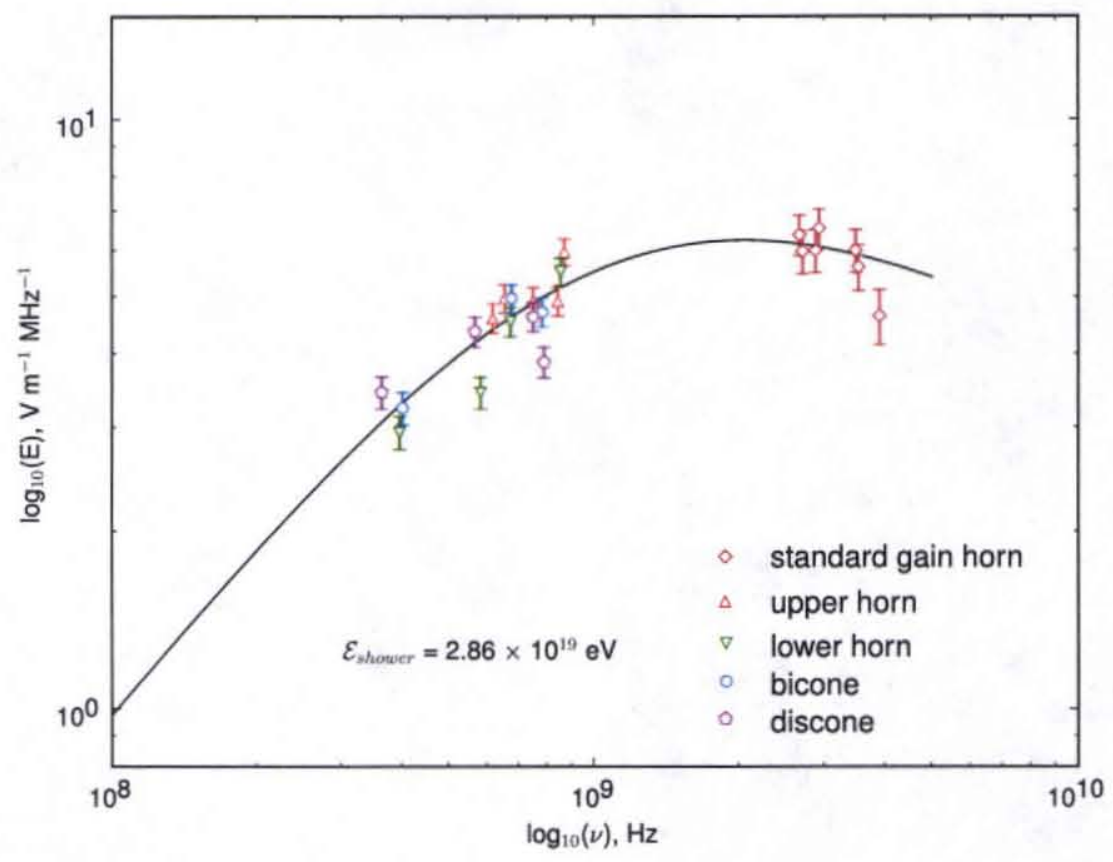

Figure 4.19: Electric field strength vs. frequency of radio Cherenkov radiation from the T486 experiment for several different antennas used, including the expected theoretical curve $[3,4]$. The lower frequency data was borrowed from Gorham et al. [5] measured with the ANITA instrument. The high frequency data came from this work with the Standard Gain Horn which illustrates the roll-off of the Cherenkov radiation from electromagnetic showers in ice with frequency.

The low-frequency data was extracted from Gorham et al. [5] measured with upper and lower Seavey horns, bicones, and discone antennas. The errors for the ANITA data are statistical, and are $\pm 40 \%$ in field strength ( $\pm 3 \mathrm{~dB}$ ). The high-frequency data was calculated from run 102 with the standard gain horn. The standard Fourier transform ${ }^{1}$ of the mea-

$$
{ }^{1} E(\omega)=\int_{-\infty}^{+\infty} E(t) e^{i \omega t} d t
$$


sured electric field was multiplied by a factor of 2 in order to agree with equation 2.2.3. The uncertainty in these data is the rms (root-mean-squared) of the field strength for the entire run which yielded approximately $\pm 50 \%$ error. The theoretical curve was generated with $\mathcal{E}_{\text {shower }}=2.86 \times 10^{19} \mathrm{eV}$ and $\nu_{0}=1.15 \mathrm{GHz}$. 


\section{Chapter 5}

\section{Conclusion}

SLAC T486 provided the confirmation of the Askaryan effect in ice: coherent radio Cherenkov emission from high-energy particle cascades in dense media is detectable. Such a validation has already been performed for two other dielectrios (salt, silica sand) which have similar radio properties as ice for observing the Askaryan effect.

In this analysis, I have demonstrated the quadratic scaling $(1.84533 \pm 0.07013)$ of Cherenkov pulse power with shower energy which indicates that the radiation is coherent over 2.6-3.95 GHz. Within rms uncertainties, the electric field strength of radio impulses received at the standard gain horn were in good agreement with simulations incorporating electrodynamics and shower properties in ice. This result illustrates the roll-off of the frequency spectrum for coherent radio Cherenkov emission.

These results provide a promising outlook for existing ultra-high energy neutrino detectors using ice as their interaction medium. The ANITA experiment has just completed its first flight observing the vast majority of the Antarctica ice for neutrino induced electromagnetic showers. Having accelerator-based experimental data confirming the electric field frequency spectrum that follows our current theory will improve efforts and sensitivity in detecting UHE neutrinos. Furthermore, SLAC T486 has illuminated what effect we can expect to see from radiation patterns penetrating the ice surface from electromagnetic cascades in the $10^{18} \rightarrow 10^{22} \mathrm{eV}$ energy regime. 


\section{Appendix A}

\section{SLAC T486 Ice Target}

As discussed in section 4.1.3, many independent measurements were made to assess the effect of surface roughness and to calibrate the slope of the carved ice surface. Since the target was carved using various hand tools, a careful examination of the surface is necessary to further understand the Cherenkov emission leaving the target.

The target was assembled using $\sim 55136 \mathrm{~kg}$ blocks of carving grade ice. With the target enclosure empty, each block was carefully stacked inside forming a large rectangular prism. The surface of the unified block of ice was then carved to create a $\sim 8^{\circ}$ slope to prevent total-internal-reflection (TIR) of the emerging radiation from the electromagnetic shower.

After carving, a $13 \times 6$ element grid was produced that contained the length, width, height, slope along the $x$-axis, slope along the $y$-axis, and the position of the ice relative to the two walls of the target enclosure along the $y$-axis. All angles were measured with a digital protractor while positional data was taken standard measuring tape. I would estimate errors in length, width, height, etc. to be within $\pm 2 \mathrm{~cm}$ and angle uncertainties to be $\pm 0.5^{\circ}$. The surface data from the ice target is summarized in the following pages. 


\begin{tabular}{|c|c|c|c|c|c|c|c|}
\hline Length $(\mathrm{cm})$ & Width $(\mathrm{cm})$ & Height $(\mathrm{cm})$ & $\theta_{x}\left(^{\circ}\right)$ & $\theta_{y}\left({ }^{\circ}\right)$ & Target Width $(\mathrm{cm})$ & +y - gap $(\mathrm{cm})$ & -y - gap $(\mathrm{cm})$ \\
\hline \hline 0 & 15.24 & 13.2 & -5.5 & -10.4 & 179.705 & 8.89 & 10.16 \\
0 & 45.72 & 13.5 & -11.8 & -1.3 & 179.705 & 8.89 & 10.16 \\
0 & 76.2 & 13.7 & -9.4 & 0 & 179.705 & 8.89 & 10.16 \\
0 & 106.68 & 13.2 & -9.4 & -3.9 & 179.705 & 8.89 & 10.16 \\
0 & 137.16 & 11.8 & -15.5 & -1 & 179.705 & 8.89 & 10.16 \\
0 & 167.64 & 13 & -15.5 & -2.3 & 179.705 & 8.89 & 10.16 \\
30.48 & 15.24 & 16.5 & -6.9 & -4.4 & 179.705 & 7.62 & 7.62 \\
30.48 & 45.72 & 16.3 & 1.1 & -4.6 & 179.705 & 7.62 & 7.62 \\
30.48 & 76.2 & 16.5 & 0.3 & 0.5 & 179.705 & 7.62 & 7.62 \\
30.48 & 106.68 & 16.2 & -4.4 & -1.8 & 179.705 & 7.62 & 7.62 \\
30.48 & 137.16 & 17 & 0.4 & 0.5 & 179.705 & 7.62 & 7.62 \\
30.48 & 167.64 & 16.4 & -3.1 & -2.7 & 179.705 & 7.62 & 7.62 \\
60.96 & 15.24 & 18.5 & -5.4 & -7.4 & 179.705 & 8.255 & 8.255 \\
60.96 & 45.72 & 17.2 & -7 & -0.58 & 179.705 & 8.255 & 8.255 \\
60.96 & 76.2 & 18.2 & -10.9 & -0.5 & 179.705 & 8.255 & 8.255 \\
60.96 & 106.68 & 18.2 & -1.4 & 1.9 & 179.705 & 8.255 & 8.255 \\
60.96 & 137.16 & 17.5 & -7 & -2 & 179.705 & 8.255 & 8.255 \\
60.96 & 167.64 & 18 & -12.2 & -11.3 & 179.705 & 8.255 & 8.255 \\
91.44 & 15.24 & 17.6 & -9.3 & -4.3 & 179.705 & 10.16 & 8.89 \\
91.44 & 45.72 & 18.8 & -6.9 & -2.1 & 179.705 & 10.16 & 8.89 \\
91.44 & 76.2 & 18.2 & -8.8 & 0.2 & 179.705 & 10.16 & 8.89 \\
91.44 & 106.68 & 18.2 & -8.7 & -1 & 179.705 & 10.16 & 8.89 \\
91.44 & 137.16 & 17.5 & -6.6 & 1.5 & 179.705 & 10.16 & 8.89 \\
91.44 & 167.64 & 19.2 & -5.5 & -5.9 & 179.705 & 10.16 & 8.89 \\
121.92 & 15.24 & 20.5 & -10.1 & -9.5 & 179.705 & 9.525 & 10.16 \\
121.92 & 45.72 & 19.9 & -5.3 & -2.5 & 179.705 & 9.525 & 10.16 \\
121.92 & 76.2 & 20 & -8.2 & -3.5 & 179.705 & 9.525 & 10.16 \\
121.92 & 106.68 & 19 & -9.3 & -1.4 & 179.705 & 9.525 & 10.16 \\
121.92 & 137.16 & 20.5 & -10 & 3.3 & 179.705 & 9.525 & 10.16 \\
121.92 & 167.64 & 21.2 & -12.3 & -2 & 179.705 & 9.525 & 10.16 \\
\hline
\end{tabular}




\begin{tabular}{|c|c|c|c|c|c|c|c|c|}
\hline Length $(\mathrm{cm})$ & Width $(\mathrm{cm})$ & Height $(\mathrm{cm})$ & $\theta_{x}\left({ }^{\circ}\right)$ & $\theta_{y}\left(^{\circ}\right)$ & Target Width $(\mathrm{cm})$ & +y - gap $(\mathrm{cm})$ & -y - gap $(\mathrm{cm})$ \\
\hline \hline 152.4 & 15.24 & 22.8 & -4.9 & -4.6 & 180.0352 & 11.43 & 6.35 \\
152.4 & 45.72 & 21.8 & -6 & 0 & 180.0352 & 11.43 & 6.35 \\
152.4 & 76.2 & 21.5 & 4 & -0.7 & 180.0352 & 11.43 & 6.35 \\
152.4 & 106.68 & 21.8 & -4.3 & 3.8 & 180.0352 & 11.43 & 6.35 \\
152.4 & 137.16 & 22.6 & -4.1 & -1.8 & 180.0352 & 11.43 & 6.35 \\
152.4 & 167.64 & 22.1 & -10.2 & 0 & 180.0352 & 11.43 & 6.35 \\
182.88 & 15.24 & 23 & -6.6 & 1.5 & 179.705 & 12.065 & 6.35 \\
182.88 & 45.72 & 22.4 & -4.4 & 3.7 & 179.705 & 12.065 & 6.35 \\
182.88 & 76.2 & 22.8 & -4.6 & -0.5 & 179.705 & 12.065 & 6.35 \\
182.88 & 106.68 & 23.6 & -4.8 & 3.1 & 179.705 & 12.065 & 6.35 \\
182.88 & 137.16 & 24 & 0.6 & 2 & 179.705 & 12.065 & 6.35 \\
182.88 & 167.64 & 24.6 & -8.8 & 4.7 & 179.705 & 12.065 & 6.35 \\
213.36 & 15.24 & 23 & -8.4 & 10.7 & 180.0352 & 13.97 & 6.35 \\
213.36 & 45.72 & 23.2 & -1.9 & 8.5 & 180.0352 & 13.97 & 6.35 \\
213.36 & 76.2 & 23.6 & -5.6 & 7.5 & 180.0352 & 13.97 & 6.35 \\
213.36 & 106.68 & 24 & -11.8 & 0.5 & 180.0352 & 13.97 & 6.35 \\
213.36 & 137.16 & 23.8 & -7.3 & 1 & 180.0352 & 13.97 & 6.35 \\
213.36 & 167.64 & 24 & -4.7 & 0.7 & 180.0352 & 13.97 & 6.35 \\
243.84 & 15.24 & 28.5 & -2 & 2 & 180.0352 & 13.6652 & 6.35 \\
243.84 & 45.72 & 27.8 & -3.9 & 11.5 & 180.0352 & 13.6652 & 6.35 \\
243.84 & 76.2 & 26.5 & -1.8 & 11.4 & 180.0352 & 13.6652 & 6.35 \\
243.84 & 106.68 & 27.2 & -2.8 & 15.1 & 180.0352 & 13.6652 & 6.35 \\
243.84 & 137.16 & 26.5 & -7.2 & 11.5 & 180.0352 & 13.6652 & 6.35 \\
243.84 & 167.64 & 26.2 & -0.7 & 8.2 & 180.0352 & 13.6652 & 6.35 \\
274.32 & 15.24 & 28 & 10 & 9 & 180.34 & 7.62 & 5.715 \\
274.32 & 45.72 & 25.5 & -7.9 & 11.6 & 180.34 & 7.62 & 5.715 \\
274.32 & 76.2 & 25 & -9.2 & 5.5 & 180.34 & 7.62 & 5.715 \\
274.32 & 106.68 & 24.2 & 7.5 & 11 & 180.34 & 7.62 & 5.715 \\
274.32 & 137.16 & 24 & 11.4 & 6 & 180.34 & 7.62 & 5.715 \\
274.32 & 167.64 & 24 & 8.8 & 13.4 & 180.34 & 7.62 & 5.715 \\
\hline
\end{tabular}




\begin{tabular}{|c|c|c|c|c|c|c|c|}
\hline Length $(\mathrm{cm})$ & Width $(\mathrm{cm})$ & Height $(\mathrm{cm})$ & $\theta_{x}\left(^{\circ}\right)$ & $\theta_{y}\left(^{\circ}\right)$ & Target Width $(\mathrm{cm})$ & $+\mathrm{y}-$ gap $(\mathrm{cm})$ & $-\mathrm{y}-\mathrm{gap}(\mathrm{cm})$ \\
\hline \hline 304.8 & 15.24 & 25 & -8.9 & 8.5 & 180.34 & 9.525 & 7.62 \\
304.8 & 45.72 & 25 & -8.2 & 8 & 180.34 & 9.525 & 7.62 \\
304.8 & 76.2 & 24 & 6.7 & 9.9 & 180.34 & 9.525 & 7.62 \\
304.8 & 106.68 & 23.4 & 8.7 & -8.9 & 180.34 & 9.525 & 7.62 \\
304.8 & 137.16 & 23.5 & -12.4 & 8.1 & 180.34 & 9.525 & 7.62 \\
304.8 & 167.64 & 23 & 2.9 & 12.6 & 180.34 & 9.525 & 7.62 \\
335.28 & 15.24 & 23.5 & -10.5 & 10.5 & 180.34 & 10.16 & 8.89 \\
335.28 & 45.72 & 21.5 & -7.2 & -2 & 180.34 & 10.16 & 8.89 \\
335.28 & 76.2 & 21.8 & -9.9 & 10.6 & 180.34 & 10.16 & 8.89 \\
335.28 & 106.68 & 21 & -8.1 & 10.7 & 180.34 & 10.16 & 8.89 \\
335.28 & 137.16 & 20.8 & -8.3 & -8 & 180.34 & 10.16 & 8.89 \\
335.28 & 167.64 & 20 & -4.2 & 14.9 & 180.34 & 10.16 & 8.89 \\
365.76 & 15.24 & 23.5 & -12.8 & 15 & 180.34 & 13.335 & 9.525 \\
365.76 & 45.72 & 21.5 & -0.3 & 5 & 180.34 & 13.335 & 9.525 \\
365.76 & 76.2 & 20 & -4.3 & 0 & 180.34 & 13.335 & 9.525 \\
365.76 & 106.68 & 30.5 & -23.5 & 0.3 & 180.34 & 13.335 & 9.525 \\
365.76 & 137.16 & 20 & -31.4 & 25 & 180.34 & 13.335 & 9.525 \\
365.76 & 167.64 & 31 & "No data" & "No data" & 180.34 & 13.335 & 9.525 \\
\hline
\end{tabular}




\section{Appendix B}

\section{Bases, Camps, \& Research Stations on the Antarctic Continent}

During preliminary analysis of the ANITA 2006-2007 Antarctic flight, a comprehensive map of the continent was developed to assess possible man-made sources of EMI. The first set of bases came from http://members.eunet.at/castaway/stations/aabases.html which gave a detailed listing of all research stations on Antarctica from the late 1940's to the present. In addition to bases and research stations, there were many expedition paths that could contribute to EMI. The main expeditions that could provide GPS data of their trek were:

1. Correne, Erasmus, \& Coetzer

2. John Wilton Davies

3. Team N2i (Novo to Inaccessibility)

4. ITASE (International Trans-Antarctic Scientific Expedition).

Another possible source of EMI could come from automated-weather-stations (AWS) that exist all over the continent to collect climate data. A detailed list of the existing AWS stations was taken from http://amrc.ssec.wisc.edu/aws.html.

The map was generated with a combination of python ${ }^{T M}$, MATPLOTLIB, and the Basemap toolkit extension for MATPLOTLIB. The topographic data was provided by the National Oceanic \& Atmospheric Administration (NOAA) with 20 m resolution (http://www.cdc.noaa.gov/Datasets/ferret/data/). The generated map, figure B uses a 
stereographic projection centered on the South Pole and displays all of the elevation data below $60^{\circ} \mathrm{S}$ in meters. The 35-day flight path of the ANITA payload is plotted in solid red, bases and research stations are point-like squares, and the AWS stations are black x's. 


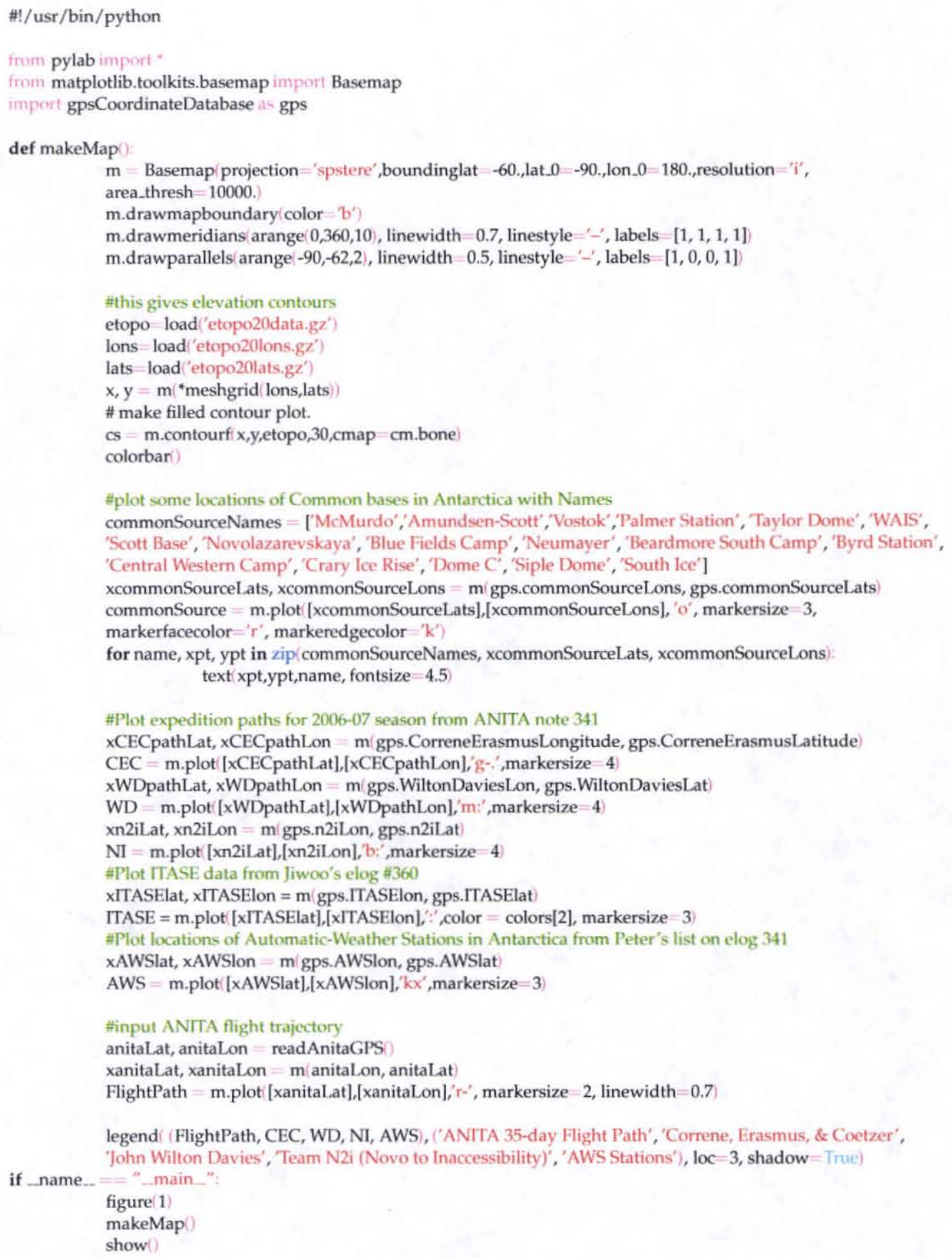




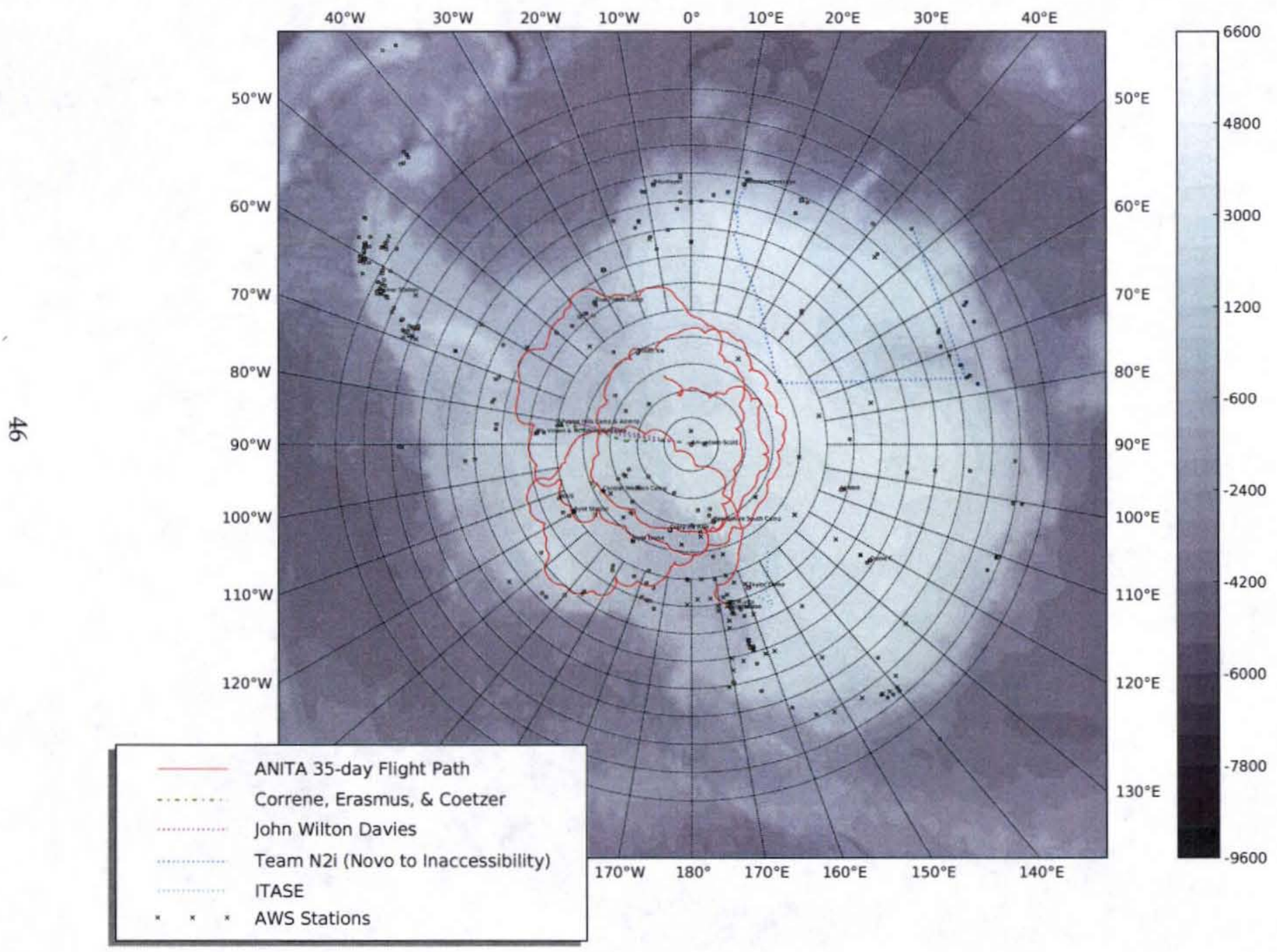




\section{Bibliography}

[1] G. S. Varner et al., Detection of Ultra High Energy Neutrinos via Coherent Radio Emission, in Proceedings of International Symposium on Detector Development for Particle, Astroparticle and Synchrotron Radiation Experiments (SNIC 2006), p. 0046, 2006.

[2] Cosmic ray energy spectrum, http://www.gnu.org/software/gsl/manual/ html \node/GNU-Free-Documentation-License .html.

[3] E. Zas, F. Halzen, and T. Stanev, Phys. Rev. D 45, 362 (1992).

[4] J. Alvarez-Muñiz, R. A. Vázquez, and E. Zas, Phys. Rev. D 62, 063001 (2000).

[5] P. W. Gorham et al., arXiv:hep-ex/0611008v2 (2007).

[6] K. Greisen, Phys. Rev. Lett. 16, 748 (1966).

[7] G. Zatsepin and V. Kuzmin, JETP Lett. 4, 78 (1966).

[8] G. A. Askaryan, JETP 14, 441 (1962).

[9] G. A. Askaryan, JETP 21, 658 (1965).

[10] P. W. Gorham et al., Phys. Rev. Lett. 93, 041101 (2004).

[11] N. G. Lehtinen, P. W. Gorham, A. R. Jacobson, and R. A. Roussel-Dupré, Phys. Rev. D 69, 013008 (2004).

[12] I. Kravchenko et al., Astropart. Phys. 20, 195 (2003).

[13] F. Halzen and D. Hooper, Phys. Rev. Lett. 97, 071101 (2006). 
[14] S. W. Barwick et al., Phys. Rev. Lett. 96, 171101 (2006).

[15] R. V. Buniy and J. P. Ralston, Phys. Rev. D 65, 016003 (2001).

[16] J. Updike, Telephone Poles and Other Poems (Knopf, 1960).

[17] E. Fermi, Z. Phys. 88, 161 (1934).

[18] F. Reines, C. L. Cowan, F. B. Harrison, A. D. McGuire, and H. W. Kruse, Phys. Rev. 117,159 (1960).

[19] G. D. Coughlan, J. E. Dodd, and B. M. Gripaios, The Ideas of Particle Physics (Cambridge University Press, 2006).

[20] G. V. Kulikov and G. B. Khristiansen, J. Exp. Theor. Phys. 8, 441 (1959).

[21] E. Waxman and J. Bahcall, Phys. Rev. D 59, 023002 (1998).

[22] M. Takeda et al., Phys. Rev. Lett. 81, 1163 (1998).

[23] D. J. Bird et al., Phys. Rev. Lett. 71, 3401 (1993).

[24] M. Ave, J. Knapp, J. Lloyd-Evans, M. Marchesini, and A. A. Watson, astroph/0112253v2 (2002).

[25] R. U. Abbasi et al., astro-ph/0208243 (2003).

[26] M. Nagano and F. Takahara, editors, The Energy Spectrum and Anisotropy of Primary Cosmic Rays at Energy $E_{0}>10^{17} \mathrm{eV}$ Obseroed in Yakutsk, Astrophysical Aspects of the Most Energetic Cosmic Rays, Proceedings of the ICRR International Symposium. Singapore: World Scientific Publishing, 1991.

[27] J. N. Bahcall and E. Waxman, Phys. Lett. B 556, 1 (2003).

[28] T. Yamamoto, The UHECR spectrum measured at the Pierre Auger Observatory and its astrophysical implications, in 30th International Cosmic Ray Conference, 2007. 
[29] D. Seckel and T. Stanev, Phys. Rev. Lett. 95, 141101 (2005).

[30] J. Alvarez-Mur̃iz and E. Zas, Phys. Lett. B 434, 396 (1998).

[31] V. S. Berezinsky and G. T. Zatsepin, Phys. Lett. 28b, 423 (1969).

[32] M. Ackermann et al., Nucl. Instrum. Meth. A556, 169 (2006).

[33] P. W. Gorham et al., Phys. Rev. E 62, 8590 (2000).

[34] P. Gorham et al., Phys. Rev. D 72, 023002 (2005).

[35] P. Mioxinović et al., Phys. Rev. D 74, 043002 (2006).

[36] S. Barwick, D. Besson, P. Gorham, and D. Saltzberg, J. Glaciol. 51, 231 (2005).

[37] P. W. Gorham, Department of Physics and Astronomy, University of Hawaii at Manoa Report No. SMEX03-0004-0019, 2003 (unpublished).

[38] J. W. Kennedy, E. R. Lusczek, G. S. Varner, and K. Liewer, ANITA Note \# 72 Antenna Channel Mapping: Gondola, SURF and TURF Phi Sector Numbering Assignment, 2006.

[39] W. R. Binns, ANITA Note \# 82 - PV I-V and I-Power calculations, 2006.

[40] G. S. Varner et al., arXiv:physics/0509023v2 (2007).

[41] G12 GPS OEM Board \& Sensor Reference Manual, Thales Navigation, 471 El Camino Real, Santa Clara, CA 95050.

[42] ADU5 Operation and Reference Manual, Thales Navigation, 471 El Camino Real, Santa Clara, CA 95050.

[43] J. D. Kraus, Antennas (McGraw-Hill, 1988).

[44] M. Ciattaglia and G. Marrocco, IEEE TRANSACTIONS ON ANTENNAS AND PROPAGATION 53, 1054 (2005). 